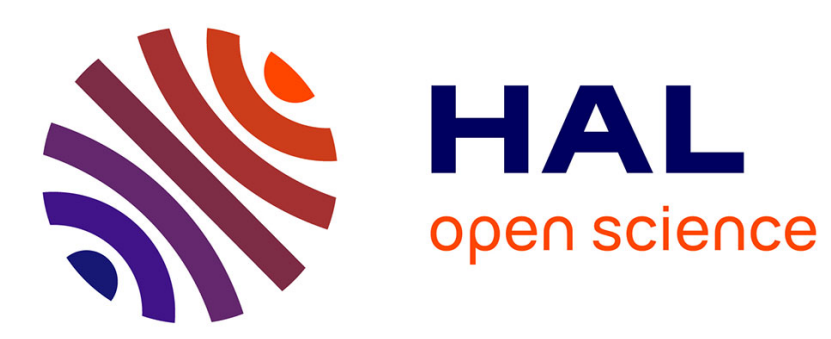

\title{
Multitype branching process with nonhomogeneous Poisson and contagious Poisson immigration
}

\author{
Landy Rabehasaina, Jae-Kyung Woo
}

\section{To cite this version:}

Landy Rabehasaina, Jae-Kyung Woo. Multitype branching process with nonhomogeneous Poisson and contagious Poisson immigration. Journal of Applied Probability, In press. hal-03144922

\author{
HAL Id: hal-03144922 \\ https://hal.science/hal-03144922
}

Submitted on 18 Feb 2021

HAL is a multi-disciplinary open access archive for the deposit and dissemination of scientific research documents, whether they are published or not. The documents may come from teaching and research institutions in France or abroad, or from public or private research centers.
L'archive ouverte pluridisciplinaire HAL, est destinée au dépôt et à la diffusion de documents scientifiques de niveau recherche, publiés ou non, émanant des établissements d'enseignement et de recherche français ou étrangers, des laboratoires publics ou privés. 


\title{
Multitype branching process with nonhomogeneous Poisson and contagious Poisson immigration
}

\section{Landy Rabehasaina and Jae-Kyung Woo}

\author{
Laboratory of Mathematics of Besançon, \\ University Bourgogne Franche Comté, \\ 16 route de Gray, 25030 Besançon cedex, France. \\ e-mail: lrabehas@univ-fcomte.fr \\ School of Risk and Actuarial Studies, \\ Australian School of Business, \\ University of New South Wales Sydney, Australia. \\ e-mail: j.k.woo@unsw.edu.au
}

\begin{abstract}
In a multitype branching process, it is assumed that immigrants arrive according to a nonhomogeneous Poisson or a contagious Poisson process (both processes are formulated as a nonhomogeneous birth process with an appropriate choice of transition intensities). We show that the normalized numbers of objects of the various types alive at time $t$ for supercritical, critical, and subcritical cases jointly converge in distribution under those two different arrival processes. Furthermore, some transient expectation results when there are only two types of particles are provided.
\end{abstract}

AMS 2000 subject classifications: Primary 60J80, 60J85; secondary 60K10, 60K25, $90 \mathrm{~B} 15$.

Keywords and phrases: Multitype branching process with immigration; Nonhomogeneous Poisson process; Contagious Poisson process; Convergence in distribution.

\section{Introduction}

Single or multitype branching processes with different stochastic assumptions on the immigration process have been applied in diverse fields in applied probability such as biology, epidemiology, and demography. For example, [27] utilized the theory of multitype branching processes in discrete time with immigration to study the joint queue length process in the different queues of a polling system in queueing theory. More generally, a network of infinite server queues may be seen as multitype Galton Watson processes with immigration, see e.g. [2]. Some actuarial applications of branching processes such as a reinsurance chain were discussed in [28, Section 7.5]. Regarding applications in biology, the recent paper [14] considered multitype branching processes with homogeneous Poisson immigration to study stress erythropoiesis, although the authors pointed out that a nonhomogeneous Poisson process (NHPP) might be more realistic in that situation. The reader is referred to [24] for a detailed discussion about the relevant literature on various types of branching processes.

In this paper, we consider a multitype branching process in which there are different types of particles, and new particles that arrive according to a NHPP or a contagious Poisson process (CPP). For immigration processes, an alternative to homogeneous Poisson process, NHPP 
and CPP are chosen for the following reasons. NHPP and CPP are within the class of nonhomogeneous birth processes, which means the intensity of event occurrence possibly varies with time (e.g. seasonality of catastrophe incidence) (NHPP) or the past state of the process (e.g. the number of previous shocks or the number of accidents incurred in the past) (CPP). As a result, these non-Poissonian processes have been widely used in various areas such as engineering, applied probability, biological science, and actuarial science. In particular, a CPP is an immigration process with a linear birth rate and is basically a homogeneous case of generalized Polya processes (GPP) studied in [35, 9, 10] for example. This process is also regarded as a contagious model which is suitable to describe the spread of an infection as proposed in [25] and the accident proneness as considered by [17], [7], and [8, Section 2.2]. Since a branching process can be used to study the dynamic network of the spread of infectious diseases, a CPP is naturally a suitable choice for the immigration arrival process to model the occurrence of contagious events due to the explanation given previously. In addition, we refer to some papers which considered GPP in other fields. For example, see [19] for a more detailed discussion on the use of GPP in the framework of a non-stationary type master equation approach in mathematical physics. As a GPP models the decreasing sequence of interarrival times depending on the number of the events in the past (of which their positive dependence is proved in [11]), it becomes a more realistic choice in modeling mortality as well as the increasingly damaging impact on an aging system as studied in [11].

The focus of our paper is to study the joint asymptotic behavior of a process representing the numbers of different types of particles alive at time $t$ when the immigration process is described by a NHPP or a CPP. These models may be interpreted differently depending on whether we are in an epidemic, actuarial, queueing or reliability setting. In an epidemic setting, the particles represent contaminated cells, the types represent their locations, and those cells can move to other locations where they can possibly contaminate other cells. In an actuarial setting, a particle may represent a certain type of claims or tasks that needs to be processed in different branches of an insurance company before being settled, or are in different stages of a reinsurance contract as explained in [28, Section 7.5]. In a queueing setting, a particle is a customer who arrives and gets served immediately in the setting of infinite server queues and after leaving the queue, is replicated into several new customers who are sent to other queues for their subsequent service. In a reliability setting, particles are interconnected parts in a system which can be damaged upon external shock arrivals and need to be repaired or are dependent line outages in a power network which may cause cascading blackouts, see [26].

Most papers in the literature consider the critical case of underlying branching mechanisms, see for e.g. [33, 12, 34, 24]. In this paper, we consider all three underlying branching mechanisms, including supercritical, critical and subcritical cases. Indeed, it is well known that in the subcritical and critical cases for a continuous time multitype Galton-Watson process, i.e. when the eigenvalue of the mean matrix of offspring does not exceed 1 , extinction is certain, whereas the survival probability in an infinite horizon is positive in the supercritical case. Depending on the modeling context, either a critical, subcritical or supercritical case may be more appropriate for modeling the dynamics of the quantity of interest. In particular, in a queueing or actuarial context where the clients or tasks will eventually exit the system, it may be more plausible to use a critical or subcritical case. For example, in the case of polling systems, the stable case corresponds to the subcritical branching process and the heavy traffic limit is studied using the near critical branching process in [30]. On the other hand, in the context of epidemiology, a supercritical case may be more appropriate to model the rapid expansion of a particular disease in the beginning of the outbreak. 
The three cases of underlying branching mechanisms exhibit different behaviors of the branching process with immigration. Concerning the immigration arrival process, particular attention in the forthcoming results is given to the case where the immigration rate increases very fast. This is a property of the GPP, as the (stochastic) arrival rate is linear with respect to the number of arrivals at the current time, so that the interarrival time decreases with respect to the failure rate order, as explained in $[11,6]$. In the NHPP, assumptions on the intensity function are such that an exponential behaviour for the latter is studied, yielding different kinds of asymptotic results. For a single type branching process with general lifetime distribution of the particles, the reader is referred to [15] and [16] which proved asymptotic results and functional central limit theorems respectively in the supercritical and subcritical cases.

In this paper, we also investigate the transient moment of the process for two types of particles branching mechanism when the renewal function associated with the arrival process is explicit. See e.g. [14] for a similar study when the particular lifetime particles have a general distribution.

The rest of the paper is organized as follows. In Section 2, a multitype branching process without/with immigration and relevant assumptions are described. Some convergence results for the distribution of a number of different types of particles at time $t$ (denoted in vector form as $N(t)$ ) under different immigration processes (NHPP, CPP) are obtained from its characteristic function $(\mathrm{CF})$. In Section 3, NHPP is assumed for the arrival process of immigrants and convergence results for $N(t)$ are given in Theorems 3 and 4, with a particular emphasis in the case when the intensity of the arrival process increases exponentially. A result in the critical case is given in Theorem 5, which agrees with the previous results in the same context in $[34,31,24]$. For the critical case, some remarks for homogeneous Poisson immigration and one dimensional branching process with immigration are provided in Remark 6. In subsections 3.1, 3.2, and 3.3, detailed proofs of Theorems 3, 4 and 5 are given. Section 4 considers a CPP for the immigration process. Asymptotic behaviors of $N(t)$ are studied in Theorem 10 in the function of the parameters of the arrival process with detailed proofs included in subsections 4.1, 4.2 and 4.3. In the proofs of Theorems 3, 4, 5 and 10, we show that, for a conveniently chosen normalizing function $g(t)$, the process $N(t) / g(t)$ converges in distribution to an identifiable limit as $t \rightarrow+\infty$ by proving that the corresponding CF converges. Finally, some transient results in scenarios when there are two types of particles in the branching process are presented in Section 5 .

Lastly, the following matrix notations will be used throughout the paper. For any matrix $M \in \mathbb{R}^{m \times n}, M^{\prime} \in \mathbb{R}^{n \times m}$ denotes its transpose. By $\left\langle u, v>=\sum_{i=1}^{k} u_{i} v_{i}\right.$ we denote the usual inner product between two vectors $u=\left(u_{1}, \ldots, u_{k}\right)^{\prime}$ and $v=\left(v_{1}, \ldots, v_{k}\right)^{\prime}$, associated with the

corresponding Euclidean norm $\|u\|=\sqrt{\sum_{i=1}^{k} u_{i}^{2}}$. We let $\mathbf{1}=(1, \ldots, 1)^{\prime}$, a vector with 1 's of an appropriate dimension, $\mathbb{R}_{+}^{k}=[0,+\infty)^{k}$ and $\mathbb{R}_{+}^{* k}=(0,+\infty)^{k}$. We also let $L^{1}\left(\mathbb{R}_{+}\right)$be the set of integrable measurable functions from $\mathbb{R}_{+}$to $\mathbb{R}$. Finally, we denote $\mathbb{N}^{*}=\{1,2, \ldots\}$ and $\mathbb{N}=\mathbb{N}^{*} \cup\{0\}$.

\section{The model}

The baseline model, a classical multitype branching process (without immigration), is described as follows. We consider a set of particles of $k$ possible types, with a type $i$ particle having exponential lifetime with mean $1 / \mu_{i}$ for $i=1, \ldots, k$, denoted by $\mathcal{E}\left(\mu_{i}\right)$ for $\mu_{i}>0$. Upon 
its death, a type $i$ particle produces $Y_{j}^{(i)}$ copies of type $j$ particles for all $j=1, \ldots, k$, where $\left(Y_{1}^{(i)}, \ldots, Y_{k}^{(i)}\right)$ is a random vector with corresponding probabilities $p_{i}(\mathbf{n})=p_{i}\left(n_{1}, \ldots, n_{k}\right)=$ $\mathbb{P}\left(Y_{j}^{(i)}=n_{j}, j=1, \ldots, k\right)$ for $\mathbf{n}=\left(n_{1}, \ldots, n_{k}\right) \in \mathbb{N}^{k}$, and generating functions defined by

$$
h_{i}(z)=h_{i}\left(z_{1}, \ldots, z_{k}\right)=\sum_{\mathbf{n} \in \mathbb{N}^{k}} p_{i}(\mathbf{n}) z_{1}^{n_{1}} \cdots z_{k}^{n_{k}}, \quad i=1, \ldots, k, \quad z \in[0,1]^{k} .
$$

In other words, $p_{i}(\mathbf{n})$ is the probability that type $i$ particle produces $n_{1}, \ldots, n_{k}$ copies of type $1, \ldots, k$ particles respectively. Then all copies evolve independently and have the same dynamics. Note that $p_{j}(0, \ldots, 0)$ is the probability that no replica is made, i.e. the probability that the particle does not produce any copies at the end of its lifetime. The mean numbers of copies from type $i$ particle are denoted by $\left(m_{i, 1}, \ldots, m_{i, k}\right)=\left(\mathbb{E}\left[Y_{1}^{(i)}\right], \ldots, \mathbb{E}\left[Y_{k}^{(i)}\right]\right)$. We suppose that $\left(Y_{1}^{(i)}, \ldots, Y_{k}^{(i)}\right)$ is not a.s. a zero vector, which guarantees that the base process does not die out immediately. We let the vector process $N^{o}(t)=\left(N_{1}^{o}(t), \ldots, N_{k}^{o}(t)\right)^{\prime}$ where $N_{j}^{o}(t)$ represents the number of $j$ type particles at time $t$. Also it is assumed that at time 0 the number of particles $N^{o}(0)$ is a random vector $I=(I(1), \ldots, I(k))^{\prime}$ with distribution

$$
I \sim \sum_{\mathbf{n} \in \mathbb{N}^{k}} p_{\mathbf{n}} \delta_{\mathbf{n}}
$$

for some probability vector $\left(p_{\mathbf{n}}\right)_{\mathbf{n} \in \mathbb{N}^{k}}$, and where $\delta_{\mathbf{n}}$ is the Dirac distribution concentrated at $\mathbf{n} \in \mathbb{N}^{k}$, and the second order moment holds

$$
\text { Assumption (A) : } \quad \mathbb{E}\left(\|I\|^{2}\right)=\sum_{j=1}^{k} \mathbb{E}\left(I(j)^{2}\right)<+\infty .
$$

A central remark is that, given $N^{o}(0)=\mathbf{n}_{0}=\left(\mathbf{n}_{0}(1), \ldots, \mathbf{n}_{0}(k)\right)^{\prime} \in \mathbb{N}^{k}, N^{o}(t)$ has the same distribution as the independent sum

$$
\sum_{j=1}^{k} \sum_{l=1}^{n_{0}(j)} N^{j, l}(t)
$$

where, for each $j=1, \ldots, k,\left(\left\{N^{j, l}(t), t \geq 0\right\}\right)_{l \in \mathbb{N}^{*}}$ are independent identically distributed (iid) copies of a generic multitype branching process $\left\{N^{j}(t)=\left(N_{1}^{j}(t), \ldots, N_{k}^{j}(t)\right), t \geq 0\right\}$ with the branching mechanism given by (1) and $N_{i}^{j}(t)$ represents the number of type $i$ particles at time $t$ produced from a type $j$ particle generated at time 0 , i.e. $N^{j}(0)$ is a vector of which $j$ th entry is 1 and 0 elsewhere. The CF of $N^{o}(t)$ is denoted by $\varphi_{t}^{o}(s):=\mathbb{E}\left[e^{<i s, N^{o}(t)>}\right]$ for $s \in \mathbb{R}^{k}$. According to [5, Chapter V], $\left\{N^{o}(t), t \geq 0\right\}$ is a continuous time multitype branching process (without immigration). Note that, in view of the representation (3), one has the following expression

$$
\varphi_{t}^{o}(s)=\mathbb{E}\left(\prod_{j=1}^{k} \varphi_{t}^{j}(s)^{I(j)}\right)=\sum_{\mathbf{n}_{0} \in \mathbb{N}^{k}} \prod_{j=1}^{k} \varphi_{t}^{j}(s)^{\mathbf{n}_{0}(j)} p_{\mathbf{n}_{0}},
$$

where $\varphi_{t}^{j}(s):=\mathbb{E}\left(\exp \left(<i s, N^{j}(t)>\right)\right)$.

We recall some useful results which will be used often in the subsequent study. First, it is convenient to introduce a $k \times k$ matrix $A=\left(a_{i j}\right)_{i, j=1, \ldots, k}$ where the $a_{i j}$ 's are defined by

$$
a_{i j}=\mu_{j}\left(m_{i j}-\mathbb{1}_{[i=j]}\right), \quad i, j=1, \ldots, k .
$$


We suppose that $A$ is regular i.e. all entries of the matrix $\exp \left(t_{0} A\right)$ are positive for some $t_{0}>0$ (see [5, Definition (10) p.202]). This entails that the largest eigenvalue $\rho$ of $A$ is with multiplicity 1. It is commonly known that we are in the subcritical case if $\rho<0$, in the critical case if $\rho=0$, and in the supercritical case if $\rho>0$. We let $u$ and $v$ be the $k \times 1$ right and left eigenvectors respectively, i.e. such that $A u=\rho u$ and $v^{\prime} A=\rho v^{\prime}$, with positive entries, and normalized in such a way that $\langle u, \mathbf{1}\rangle=1$ and $\langle u, v\rangle=1$. Then, in [5, Theorem 1 p.209] it was shown that $\left\{\left\langle u, N^{o}(t) e^{-\rho t}\right\rangle, t \geq 0\right\}$ is a martingale when $N^{o}(0)$ is a constant $\mathbf{n}_{0} \in \mathbb{N}^{k}$. In the case when $N^{o}(0)=I$ given by (2), it is clear that this property still holds because Assumption (A) in particular implies that $I$ is integrable. Finally, we will suppose that the process is non singular, i.e. that for all $i=1, \ldots, k, p_{i}(\mathbf{n})$ in (1) is not in the form $p_{i}(\mathbf{n})=\mathbb{1}_{\left[n_{i}=1, n_{j}=0, j \neq i\right]}$ for all $i, j=1, \ldots, k$, meaning that each particle does not produce exactly one offspring.

From [5, Theorem 2 p.206] the a.s. asymptotic behavior of $N^{o}(t)$ as $t \rightarrow+\infty$ is given in the following lemma under the assumptions described previously.

Lemma 1. There exists a non-negative random variable (rv) $W$ such that

$$
\lim _{t \rightarrow+\infty} N^{o}(t) e^{-\rho t}=W v, \quad \text { a.s. }
$$

Note that the CF of $W$ is given by

$$
\varphi_{W}(x)=\mathbb{E}\left[e^{i x W}\right]=\sum_{\mathbf{n}_{0} \in \mathbb{N}^{k}} \mathbb{E}\left[e^{i x W} \mid N^{o}(0)=\mathbf{n}_{0}\right] p_{\mathbf{n}_{0}}, \quad x \in \mathbb{R} .
$$

Because of (3), one has

$$
\mathbb{E}\left[e^{i x W} \mid N^{o}(0)=\mathbf{n}_{0}\right]=\mathbb{E}\left[e^{i x \sum_{j=1}^{k} \sum_{l=1}^{\mathbf{n}_{0}(j)} W^{j, l}}\right]=\prod_{j=1}^{k} \varphi_{j}(x)^{\mathbf{n}_{0}(j)},
$$

where $W^{j, l}$ is the a.s. limit of $N^{j, l}(t) e^{-\rho t}$ as $t \rightarrow+\infty$. For each $j=1, \ldots, k,\left(W^{j, l}\right)_{l \in \mathbb{N}}$ is an iid sequence having a CF denoted by $\varphi_{j}(x)=\mathbb{E}\left(e^{i x W^{j, l}}\right)$, and the $W^{j, l}, j=1, \ldots, k, l=1, \ldots, \mathbf{n}_{0}(j)$ are independent. The vector $\varphi(x):=\left(\varphi_{1}(x), \ldots, \varphi_{k}(x)\right)$ is in general not explicit but satisfies a particular integral equation (see [5, Eq.(28) p.206] for details). Finally, the CF of $W$ is then given as a function of this vector $\varphi(x)$

$$
\varphi_{W}(x)=\sum_{\mathbf{n}_{0} \in \mathbb{N}^{k}} \prod_{j=1}^{k} \varphi_{j}(x)^{\mathbf{n}_{0}(j)} p_{\mathbf{n}_{0}}=\mathbb{E}\left(\prod_{j=1}^{k} \varphi_{j}(x)^{I(j)}\right), \quad x \in \mathbb{R} .
$$

We then move on to a multitype branching process with immigration which is the central stochastic process studied in this paper. Let us consider that new particles (immigrants) arrive at time $T_{i}, i \geq 1$ according to a random vector $I_{i}=\left(I_{i}(1), \ldots, I_{i}(k)\right)^{\prime}$ having the same distribution (2) as $I$. Then it evolves according to the branching mechanism described at the beginning of this section. We assume that the immigration sequence $\left(I_{i}\right)_{i \in \mathbb{N}}$ is iid. The vector process $N(t)=\left(N_{1}(t), \ldots, N_{k}(t)\right)^{\prime}$ represents the number of each type of particles at time $t$ defined as:

$$
N(t)=\sum_{i=1}^{S(t)} N^{o, i}\left(t-T_{i}\right), \quad t \geq 0
$$


where $\left\{N^{o, i}(t), t \geq 0\right\}_{i \in \mathbb{N}}$ are iid copies of $\left\{N^{o}(t), t \geq 0\right\}$ with $N^{o, i}(0)=I_{i}$. The process $\{S(t), t \geq 0\}$ with $S(0)=0$ is the arrival process for new particles associated with a nondecreasing sequence $\left(T_{i}\right)_{i \in \mathbb{N}}$ with $T_{0}=0$ which represents the arrival time of the $i$ th particle, and interarrival times $\left(T_{i}-T_{i-1}\right)_{i \in \mathbb{N}^{*}}$. In other words, $N^{o, i}\left(t-T_{i}\right)$ is a vector of the number of particles in each system at time $t$ generated from the $I_{i}(j)$ particles of type $j, j=1, \ldots, k$, arrived at $T_{i}$. Also, an underlying assumption is that $N_{j}^{o, i}(t)=0$ when $t<0$ for $j=1, \ldots, k$, $\{S(t), t \geq 0\}$ and $\left\{N^{o, i}(t), t \geq 0\right\}, i \in \mathbb{N}$, are processes independent of each other. Hence, $N(t)$ is a continuous time multitype branching process with immigration given by the process $\{S(t), t \geq 0\}$.

\section{Immigration modelled by a nonhomogeneous Poisson process (NHPP)}

We assume in this section that $\{S(t), t \geq 0\}$ is a NHPP with intensity $\lambda(t)>0$ for $t \geq 0$ being locally integrable, and set $\Lambda(t):=\int_{0}^{t} \lambda(y) d y$ for $t \geq 0$.

To study the asymptotic behavior of $N(t)$ in (7) when $t \rightarrow+\infty$, we first need the CF of $N(t)$. The following result is an easy extension of [12, Equation (2)]; see also [24, Theorem 1] for a similar result that concerns the probability generating function of $N(t)$.

Lemma 2. The CF of $N(t)$ in (7) admits the following expression

$$
\varphi_{t}(s)=\mathbb{E}\left[e^{<i s, N(t)>}\right]=\exp \left\{\int_{0}^{t}\left[\varphi_{t-x}^{o}(s)-1\right] \lambda(x) d x\right\}=\exp \left\{\int_{0}^{t}\left[\varphi_{x}^{o}(s)-1\right] \lambda(t-x) d x\right\},
$$

for all $s \in \mathbb{R}^{k}$.

Proof. Given $S(t)=n,\left(T_{1}, \ldots, T_{n}\right)$ are distributed as the ordered statistics $\left(U_{(1)}, \ldots, U_{(n)}\right)$ with $\left(U_{1}, \ldots, U_{n}\right)$ being independent with density $\frac{\lambda(y)}{\Lambda(t)} \mathbb{1}_{[0, t]}(y)$, one finds

$$
\varphi_{t}(s)=\sum_{n=0}^{\infty} \mathbb{E}\left[\exp \left\{<i s, \sum_{l=1}^{n} N^{o, l}\left(t-U_{(l)}\right)>\right\}\right] e^{-\Lambda(t)} \frac{(\Lambda(t))^{n}}{n !} .
$$

Since $\sum_{l=1}^{n} N^{o, l}\left(t-U_{(l)}\right)=\sum_{l=1}^{n} N^{o, l}\left(t-U_{l}\right)$ and by independence of $\left(U_{1}, \ldots, U_{n}\right)$ and the process $\left\{N^{o, l}(t), t \geq 0\right\}$, one obtains

$$
\varphi_{t}(s)=\sum_{n=0}^{\infty}\left\{\frac{1}{\Lambda(t)} \int_{0}^{t} \mathbb{E}\left[\exp \left(<i s, N^{o}(t-y)>\right)\right] \lambda(y) d y\right\}^{n} e^{-\Lambda(t)} \frac{(\Lambda(t))^{n}}{n !} .
$$

A change of variable $x:=t-y$ concludes the proof.

The following results show that the normalized process converges towards different limits depending on the assumptions regarding the intensity of the arrival process.

Theorem 3. Suppose that the intensity $\lambda(t)$ of the NHPP $\{S(t), t \geq 0\}$ satisfies that $e^{-\rho t} \lambda(t)$ is integrable. Then

$$
e^{-\rho t} N(t) \stackrel{\mathcal{D}}{\longrightarrow} \int_{0}^{\infty} e^{-\rho z} d \mathcal{Y}_{z}^{W}, \quad t \rightarrow+\infty
$$

where $\left\{\mathcal{Y}_{t}^{W}, t \geq 0\right\}$ is a nonhomogeneous compound Poisson process with intensity $\lambda(y)$ and jumps distributed as $W v$. 
Proof. The proof is given in Section 3.1

Although Theorem 3 is valid when the eigenvalue $\rho$ has any sign, it is especially interesting in the supercritical case $\rho>0$, as $e^{-\rho t} \lambda(t) \in L^{1}\left(\mathbb{R}_{+}\right)$implies that the intensity $\lambda(t)$ can, for example, grow exponentially as $e^{\delta t}$ for $0 \leq \delta<\rho$. However Theorem 3 becomes less interesting in the critical case $\rho=0$ or subcritical case $\rho<0$, as the condition $e^{-\rho t} \lambda(t) \in L^{1}\left(\mathbb{R}_{+}\right)$roughly means that the intensity tends to 0 potentially very fast. Hence, the following supplementary results show how the renormalized process $N(t)$ converges in distribution or in probability

- when the intensity grows exponentially in the critical or subcritical case,

- when the intensity grows exponentially as $e^{\delta t}$ with $\delta \geq \rho$ in the supercritical case, complementing Theorem 3.

Theorem 4. Let us suppose that the intensity $\lambda(t)$ of the NHPP $\{S(t), t \geq 0\}$ satisfies $\lambda(t) \sim \lambda_{\infty} e^{\delta t}$ as $t \rightarrow+\infty$ for some $\delta \geq 0$ and $\lambda_{\infty}>0$. Then the following convergences hold as $t \rightarrow+\infty$ :

$$
\begin{aligned}
N(t) & \stackrel{\mathcal{D}}{\longrightarrow} \quad \nu \quad \text { if } \rho<0 \text { and } \delta=0, \\
e^{-\delta t} N(t) & \stackrel{\mathbb{P}}{\longrightarrow} \lambda_{\infty}(\delta \mathrm{Id}-A)^{-1} \mathbb{E}(I) \quad \text { if }[\rho \leq 0 \text { and } \delta>0] \text { or }[\rho>0 \text { and } \delta>\rho], \\
e^{-\delta t} \frac{N(t)}{t} & \stackrel{\mathbb{P}}{\longrightarrow} \lambda_{\infty} u v^{\prime} \mathbb{E}(I) \quad \text { if } \rho=\delta>0
\end{aligned}
$$

where Id is the identity matrix and $\nu$ is a distribution on $\mathbb{R}_{+}^{k}$ with CF given by

$$
\int_{\mathbb{R}_{+}^{k}} e^{<i s, x>} \nu(d x)=\exp \left\{\lambda_{\infty} \int_{0}^{+\infty}\left[\varphi_{y}^{o}(s)-1\right] d y\right\}, \quad s \in \mathbb{R}^{k}
$$

Proof. The proof is presented in Section 3.2.

In the following, we are especially interested in the particular critical case $\rho=0$. From Theorems 3 and 4: we have that, if $\lambda(t)$ is integrable then $N(t)$ converges in distribution to $\lim _{t \rightarrow+\infty} \mathcal{Y}_{t}^{W}$ in (9), and if $\lambda(t) \sim \lambda_{\infty} e^{\delta t}$ with $\delta>0$ then one has the convergence in probability of $e^{-\delta t} N(t)$ in (11). We note that an intermediary case is worth exploring when $\lambda(t)$ does not have an explosive behaviour and roughly speaking, does not converge to 0 . This is the case if the associated Cesaro $\operatorname{limit}_{t \rightarrow \infty} \Lambda(t) / t=\lambda_{\infty}$ exists, hence some additional convergence result may be obtained. Before detailing this convergence result, we introduce the following quantities:

$$
\begin{aligned}
Q & :=\frac{1}{2} \sum_{i, \ell, n=1}^{k} \frac{\partial^{2} h_{i}}{\partial z_{\ell} \partial z_{n}}(1, \ldots, 1) u_{\ell} u_{n} v_{i}, \\
\beta_{j} & :=\left(\sum_{\ell=1}^{k} \mu_{\ell}^{-1} u_{\ell} v_{\ell}\right) \frac{u_{j}}{Q}, \quad j=1, \ldots, k, \\
\bar{\beta} & :=\sum_{j=1}^{k} \mathbb{E}(I(j)) \beta_{j}, \\
c & :=\frac{\left(\sum_{\ell=1}^{k} \mu_{\ell}^{-1} u_{\ell} v_{\ell}\right)^{2}}{Q}
\end{aligned}
$$


where we recall that $h_{i}(z)=h_{i}\left(z_{1}, \ldots, z_{k}\right)$ is the generating function associated with $\left(p_{i}(\mathbf{n})\right)_{\mathbf{n} \in \mathbb{N}^{k}}$ given in (1). We note that the assumption that $\left(Y_{1}^{(i)}, \ldots, Y_{k}^{(i)}\right)$ is not zero for some $i \in\{1, \ldots, k\}$ entails that $Q$ is positive. The following result holds.

Theorem 5. Let us assume that the moments of all orders of the random vector $\left(Y_{1}^{(i)}, \ldots, Y_{k}^{(i)}\right)$ exist for all $i=1, \ldots, k$ and the intensity admits a Cesaro finite limit $\lambda_{\infty}=\lim _{t \rightarrow \infty} \Lambda(t) / t>0$. When $\rho=0$ (critical case), one has the convergence in distribution as

$$
\frac{N(t)}{t} \stackrel{\mathcal{D}}{\longrightarrow} \mathcal{Z} v \otimes \mu^{-1}, \quad t \rightarrow+\infty
$$

where $\mathcal{Z}$ is a rv distributed as $\Gamma\left(\lambda_{\infty} \bar{\beta}, c\right)$ with $v \otimes \mu^{-1}=\left(v_{1} \mu_{1}^{-1}, \ldots, v_{k} \mu_{k}^{-1}\right), \bar{\beta}$ and $c$ given by (14) and (15) respectively. Here, $\Gamma(\alpha, \theta)$ denotes the gamma distribution with a shape parameter $\alpha$ and a rate parameter $\theta$.

Proof. The proof is presented in Section 3.3.

Thus, it turns out that, in the critical case the support of the limits (9), (11) and (16) are respectively included in the positive half line spanned by $v,(\delta \operatorname{Id}-A)^{-1} \mathbb{E}(I)$ and $v \otimes \mu^{-1}$. It is worthwhile to point out that all three results concern the critical case, but with different assumptions on the intensity function.

Remark 6. When the intensity $\lambda(t)$ is a constant value $\lambda$, Theorem 5 is the particular case of [34, Theorem 2] which considers general interarrival times, with a slight change of notation (in that reference $\mu_{i}$ stands for the mean lifetime of a type $i$ particle, as opposed to $\mu_{i}^{-1}$ in this paper). See also [31, Theorem 1] for a similar result. When $\lambda(t)$ converges to some limit $\lambda_{\infty}$, it converges towards the same limit in the sense of Cesaro and the limit in distribution (16) corresponds to [24, Theorem 8]. However, in this paper we introduce a different approach to the proof of Theorem 5 (given in Section 3.3). In contrary to [34] which proved the result by showing that the joint moments of $N(t) / t$ converge, our approach does not require renewal arguments and relevant results. In particular, we start directly with the CF (8) which is expressed handily in Lemma 2 and study its convergence. A similar approach was adopted in [24] although the authors in [24] started the proof from a uniform estimate from [29] for the probability generating function of $\left\{N^{o}(t), t \geq 0\right\}$.

Remark 7. From (52) in the proof of Theorem 5, the limiting distribution of (16) admits a similar integral form as the right-hand side of (9) which is available by applying Campbell's formula (the details are given in the beginning of Section 3.1). Indeed, one can check the equality in distribution of $\mathcal{Z} v \otimes \mu^{-1}$ and $\int_{0}^{+\infty} e^{-t} d \mathcal{Y}_{t}^{c}$ where $\left\{\mathcal{Y}_{t}^{c}, t \geq 0\right\}$ is a compound Poisson process with intensity $\lambda_{\infty} \bar{\beta}$ and jumps distributed as $\chi v \otimes \mu^{-1}$ with $\chi \sim \mathcal{E}(c)$.

We now proceed to the proofs of Theorems 3,4 and 5 .

\subsection{Proof of Theorem 3}

We start from the CF in (8), which entails that the CF of $e^{-\rho t} N(t)$ is given by

$$
\varphi_{t}\left(s e^{-\rho t}\right)=\exp \left\{\int_{0}^{t}\left[\varphi_{x}^{o}\left(s e^{-\rho t}\right)-1\right] \lambda(t-x) d x\right\}, \quad s \in \mathbb{R}^{k} .
$$


The main difficulty in the proof is to show the following convergence:

$$
\int_{0}^{t}\left[\varphi_{x}^{o}\left(s e^{-\rho t}\right)-1\right] \lambda(t-x) d x \longrightarrow \int_{0}^{+\infty}\left[\varphi_{W v}\left(s e^{-\rho x}\right)-1\right] \lambda(x) d x, \quad t \rightarrow+\infty, s \in \mathbb{R}^{k},
$$

where $\varphi_{W v}$ is the CF of $W v$. By Campbell's formula (see [20, Formula (2.9), Theorem 2.7 p.41] or [18, Exercise 8.6.3.11 p.492]), $\exp \left\{\int_{0}^{+\infty}\left[\varphi_{W v}\left(s e^{-\rho y}\right)-1\right] \lambda(y) d y\right\}$ is the CF of $\int_{0}^{\infty} e^{-\rho z} d \mathcal{Y}_{z}^{W}$, where $\left\{\mathcal{Y}_{t}^{W}, t \geq 0\right\}$ is a nonhomogeneous compound Poisson process with intensity $\lambda(y)$ and jumps distributed as $W v$. Hence one has the convergence in distribution of $e^{-\rho t} N(t)$ towards $\int_{0}^{\infty} e^{-\rho z} d \mathcal{Y}_{z}^{W}$ if (17) holds. This proves (9).

In order to prove (17) we need to exploit the convergence $N^{o}(y) e^{-\rho y} \longrightarrow W v$ a.s. as $y \rightarrow+\infty$ given in Lemma 1. Studying (17) is equivalent to analyzing the limit as $t \rightarrow+\infty$ of

$$
Q_{t}:=\int_{0}^{t}\left[\varphi_{t-x}^{o}\left(s e^{-\rho t}\right)-1\right] \lambda(x) d x=\int_{0}^{t}\left\{\mathbb{E}\left[\exp \left(<i s, N^{o}(t-x) e^{-\rho t}>\right)\right]-1\right\} \lambda(x) d x .
$$

That is, $Q_{t}$ may be expressed as

$$
Q_{t}:=Q_{1, t}+Q_{2, t}
$$

where

$$
\begin{aligned}
Q_{1, t} & :=\int_{0}^{t}\left\{\mathbb{E}\left[\exp \left(<i s, \frac{N^{o}(t-x)}{e^{\rho(t-x)}} e^{-\rho x}>\right)-\exp \left(<i s, W v e^{-\rho x}>\right)\right]\right\} \lambda(x) d x \\
Q_{2, t} & :=\int_{0}^{t}\left\{\mathbb{E}\left[\exp \left(<i s, W v e^{-\rho x}>\right)\right]-1\right\} \lambda(x) d x
\end{aligned}
$$

We then separately examine the limits of (20) and (21). In the end, it will be shown that (20) tends to 0 and (21) tends to the right-hand side of (17).

Limit of $Q_{1, t}$ in (20) as $t \rightarrow+\infty$. We shall utilize the following basic inequality in the subsequent proof:

$$
\left|e^{i a}-e^{i b}\right| \leq|a-b|, \quad a \in \mathbb{R}, b \in \mathbb{R},
$$

and also we have $\left|e^{i a}-e^{i b}\right| \leq 2$. Hence $\left|e^{i a}-e^{i b}\right| \leq|a-b| \wedge 2$ for $a \in \mathbb{R}$ and $b \in \mathbb{R}$. We then deduce that

$$
\begin{array}{rl}
\left|Q_{1, t}\right| \leq \int_{0}^{t} & \mathbb{E}\left[\left|<s, \frac{N^{o}(t-x)}{e^{\rho(t-x)}} e^{-\rho x}>-<s, W v e^{-\rho x}>\right| \wedge 2\right] \lambda(x) d x \\
\quad=\int_{0}^{\infty} \mathbb{1}_{[0 \leq x \leq t]} \mathbb{E}\left[\left|<s, \frac{N^{o}(t-x)}{e^{\rho(t-x)}} e^{-\rho x}>-<s, W v e^{-\rho x}>\right| \wedge 2\right] \lambda(x) d x .
\end{array}
$$

By the dominated convergence theorem, we will show in the following that (23) tends to zero as $t \rightarrow+\infty$. From the pointwise convergence as $t \rightarrow+\infty$ in Lemma 1 with the help of the dominated convergence theorem, one finds that the integrand goes to zero i.e.

$$
\mathbb{1}_{[0 \leq x \leq t]} \mathbb{E}\left[\left|e^{-\rho x}<s, \frac{N^{o}(t-x)}{e^{\rho(t-x)}}>-e^{-\rho x}<s, W v>\right| \wedge 2\right] \lambda(x) \longrightarrow 0, \quad t \rightarrow \infty, \forall x \geq 0 .
$$

We now find an upper bound function $f(x) \geq 0$ of this integrand such that $\int_{0}^{+\infty} f(x) d x<+\infty$. Recall that $u$ is an eigenvector with positive entries $u_{i}$ for $i=1, \ldots, k$ such that $A u=\rho u$ 
(where the elements of the matrix $A$ are defined in (5)). Since $u_{i}>0$ for all $i$, there exists some constant $\kappa>0$ which is large enough satisfying

$$
0 \leq\left|s_{j}\right| \leq \kappa u_{j}, \quad \forall j=1, \ldots, k,
$$

where we recall that the vector $s=\left(s_{1}, \ldots, s_{k}\right)$ is fixed. For example, $\kappa$ can be chosen as $\max _{j=1, \ldots, k}\left|s_{j}\right| / u_{j}$. Also, note that $\mathbb{E}[(X+Y) \wedge 2] \leq(\mathbb{E}[X]+\mathbb{E}[Y]) \wedge 2$ for nonnegative random variables $X$ and $Y$. Using these results and the martingale property of $\left\{\left\langle u, N^{o}(t) e^{-\rho t}\right\rangle, t \geq\right.$ $0\}$, also denoting $|s|:=\left(\left|s_{1}\right|, \ldots,\left|s_{k}\right|\right)$, we conclude that the integrand is bounded as

$$
\begin{aligned}
& \mathbb{1}_{[0 \leq x \leq t]}\left\{\left(e^{-\rho x} \mathbb{E}\left[\left|<s, \frac{N^{o}(t-x)}{e^{\rho(t-x)}}>\right|\right]+e^{-\rho x} \mathbb{E}[|<s, W v>|]\right) \wedge 2\right\} \lambda(x) \\
& \leq \mathbb{1}_{[0 \leq x \leq t]}\left\{\left(e^{-\rho x} \mathbb{E}\left[<|s|, \frac{N^{o}(t-x)}{e^{\rho(t-x)}}>\right]+e^{-\rho x} \mathbb{E}[<|s|, W v>]\right) \wedge 2\right\} \lambda(x) \\
& \leq \mathbb{1}_{[0 \leq x \leq t]}\left\{\left(e^{-\rho x} \kappa \mathbb{E}\left[<u, \frac{N^{o}(t-x)}{e^{\rho(t-x)}}>\right]+e^{-\rho x} \mathbb{E}[<|s|, W v>]\right) \wedge 2\right\} \lambda(x),
\end{aligned}
$$

where the first equality is due to the fact that $\frac{N^{o}(t-x)}{e^{\rho(t-x)}}$ and $W v$ have nonnegative entries, and the last inequality is due to (24). The first expectation in (25) is $\mathbb{E}\left[<u, N^{o}(0) / e^{\rho \times 0}>\right]$ because of the martingale property and in turn, it is equal to $<u, \mathbb{E}(I)>$ because of $N^{o}(0)=I$. The second expectation is some finite constant. Therefore we conclude that, for some constants $K>0$ and $K^{*}>0,(25)$ is bounded as

$$
\begin{aligned}
& \mathbb{1}_{[0 \leq x \leq t]}\left\{\left(e^{-\rho x} \kappa \mathbb{E}\left[<u, \frac{N^{o}(t-x)}{e^{\rho(t-x)}}>\right]+e^{-\rho x} \mathbb{E}[<|s|, W v>]\right) \wedge 2\right\} \lambda(x) \\
& \quad=\mathbb{1}_{[0 \leq x \leq t]}\left[\left(\kappa<u, \mathbb{E}(I)>e^{-\rho x}+K e^{-\rho x}\right) \wedge 2\right] \lambda(x) \leq K^{*} e^{-\rho x} \lambda(x):=f(x) .
\end{aligned}
$$

It is now shown that the integrand in (23) tends to 0 as $t \rightarrow+\infty$ for a fixed $x$ and is dominated by the function $f(x)$ which is integrable by assumption. Therefore, by the dominated convergence theorem we conclude that (23) goes to 0 as $t \rightarrow \infty$, which implies that $Q_{1, t}$ in (20) verifies $\lim _{t \rightarrow \infty} Q_{1, t}=0$.

Limit of $Q_{2, t}$ in (21) as $t \rightarrow+\infty$. Let us show that $\left|\left\{\varphi_{W v}\left(s e^{-\rho x}\right)-1\right\} \lambda(x)\right|$ is upper bounded by some integrable function. With the help of (22), the following inequality holds:

$$
\left|\exp \left(<i s, W v e^{-\rho x}>\right)-1\right| \leq\left|<s, W v e^{-\rho x}>\right| \leq e^{-\rho x}<|s|, W v>.
$$

We then arrive at the following bound

$$
\left|\left\{\varphi_{W v}\left(s e^{-\rho x}\right)-1\right\} \lambda(x)\right|=\left|\left\{\mathbb{E}\left[\exp \left(<i s, W v e^{-\rho x}>\right)\right]-1\right\} \lambda(x)\right| \leq e^{-\rho x} \lambda(x) \mathbb{E}[<|s|, W v>],
$$

which indeed is integrable by the integrability assumption for $e^{-\rho x} \lambda(x)$. Then by the dominated convergence theorem one finds

$$
Q_{2, t} \longrightarrow \int_{0}^{+\infty}\left\{\varphi_{W v}\left(s e^{-\rho x}\right)-1\right\} \lambda(x) d x, \quad t \rightarrow \infty
$$

Hence, combining the limits of $Q_{1, t}$ and $Q_{2, t},(17)$ is proved. Consequently, this completes the proof. 


\subsection{Proof of Theorem 4}

Proving the limit of $N(t)$ in (10) as $t \rightarrow+\infty$. First, we recall that $\rho<0$ and $\delta=0$, and the intensity satisfies $\lim _{t \rightarrow \infty} \lambda(t)=\lambda_{\infty}$. We proceed to show that $\int_{0}^{t}\left[\varphi_{x}^{o}(s)-1\right] \lambda(t-x) d x$ in Lemma 2 converges to $\lambda_{\infty} \int_{0}^{\infty}\left[\varphi_{x}^{o}(s)-1\right] d x$ as $t \rightarrow+\infty$ by a dominated convergence argument. Let $A_{\lambda} \geq 0$ be large enough such that $C_{\lambda}:=\sup _{y \geq A_{\lambda}} \lambda(y)$ is finite, and $t \geq A_{\lambda}$. We start by writing

$$
\begin{aligned}
& \int_{0}^{t}[\left.\varphi_{x}^{o}(s)-1\right] \lambda(t-x) d x=\int_{0}^{\infty} \mathbb{1}_{[0 \leq x \leq t]}\left[\varphi_{x}^{o}(s)-1\right] \lambda(t-x) d x \\
& \quad=\int_{0}^{\infty} \mathbb{1}_{\left[0 \leq x \leq t-A_{\lambda}\right]}\left[\varphi_{x}^{o}(s)-1\right] \lambda(t-x) d x+\int_{0}^{\infty} \mathbb{1}_{\left[t-A_{\lambda}<x \leq t\right]}\left[\varphi_{x}^{o}(s)-1\right] \lambda(t-x) d x .
\end{aligned}
$$

Let us study each term on the right-hand side of (26). Using the inequality in (24), one finds

$$
\begin{array}{r}
\left|\varphi_{x}^{o}(s)-1\right|=\left|\mathbb{E}\left[e^{<i s, N^{o}(x)>}\right]-1\right| \leq \mathbb{E}\left[\left|e^{<i s, N^{o}(x)>}-1\right|\right] \leq \mathbb{E}\left[\left|<s, N^{o}(x)>\right|\right] \\
\leq \mathbb{E}\left[<|s|, N^{o}(x)>\right] \leq \kappa \mathbb{E}\left[<u, N^{o}(x)>\right]=\kappa e^{\rho x} \mathbb{E}\left[<u, N^{o}(x) e^{-\rho x}>\right] \\
=\kappa e^{\rho x} \mathbb{E}\left[<u, N^{o}(0)>\right]=\kappa e^{\rho x}<u, \mathbb{E}(I)>
\end{array}
$$

where we recall that $\kappa=\max _{j=1, \ldots, k}\left|s_{j}\right| / u_{j}$ for example. Since $\lambda($.$) is locally integrable (so$ that $\int_{0}^{A_{\lambda}} \lambda(x) d x$ is finite), and $e^{\rho x} \leq e^{\rho\left(t-A_{\lambda}\right)}$ for $t-A_{\lambda} \leq x$ and $\rho<0$, the second term on the right-hand side of (26) thus verifies

$$
\begin{gathered}
\left|\int_{0}^{\infty} \mathbb{1}_{\left[t-A_{\lambda}<x \leq t\right]}\left[\varphi_{x}^{o}(s)-1\right] \lambda(t-x) d x\right| \leq \int_{0}^{\infty} \mathbb{1}_{\left[t-A_{\lambda}<x \leq t\right]}\left|\varphi_{x}^{o}(s)-1\right| \lambda(t-x) d x \\
\leq \kappa<u, \mathbb{E}(I)>\int_{0}^{\infty} \mathbb{1}_{\left[t-A_{\lambda}<x \leq t\right]} e^{\rho x} \lambda(t-x) d x \\
\leq \kappa<u, \mathbb{E}(I)>e^{\rho\left(t-A_{\lambda}\right)} \int_{0}^{\infty} \mathbb{1}_{\left[t-A_{\lambda}<x \leq t\right]} \lambda(t-x) d x \\
\quad=\kappa<u, \mathbb{E}(I)>e^{\rho\left(t-A_{\lambda}\right)} \int_{0}^{A_{\lambda}} \lambda(x) d x \rightarrow 0, \quad t \rightarrow \infty, t \geq A_{\lambda} .
\end{gathered}
$$

Concerning the first term on the right-hand side of (26), from (27) and the definition of $C_{\lambda}$ we find that

$$
\begin{aligned}
\mathbb{1}_{\left[0 \leq x \leq t-A_{\lambda}\right]}\left|\varphi_{x}^{o}(s)-1\right| \lambda(t-x) \leq \mathbb{1}_{\left[0 \leq x \leq t-A_{\lambda}\right]} \kappa e^{\rho x}<u, \mathbb{E}(I)>\lambda(t-x) & \\
& \leq C_{\lambda} \kappa e^{\rho x}<u, \mathbb{E}(I)>
\end{aligned}
$$

Since $\rho<0$, the right-hand side of (29) is integrable, and $\lim _{t \rightarrow+\infty} \mathbb{1}_{\left[0 \leq x \leq t-A_{\lambda}\right]}\left[\varphi_{x}^{o}(s)-1\right] \lambda(t-$ $x)=\left[\varphi_{x}^{o}(s)-1\right] \lambda_{\infty}$, the dominated convergence theorem entails that the first term on the right-hand side of (26) converges to $\lambda_{\infty} \int_{0}^{\infty}\left[\varphi_{x}^{o}(s)-1\right] d x$ as $t \rightarrow+\infty$. Hence, combining with the limit obtained in (28), we deduce that the limit of the left-hand side of (26) as $t \rightarrow \infty$ is $\lambda_{\infty} \int_{0}^{\infty}\left[\varphi_{x}^{o}(s)-1\right] d x$. We now argue that the function $s \mapsto \exp \left(\lambda_{\infty} \int_{0}^{\infty}\left[\varphi_{x}^{o}(s)-1\right] d x\right)$ is continuous in a neighborhood of $0 \in \mathbb{R}^{k}$ such as $s \in(-1,1)^{k}$, which by Lévy's continuity theorem implies that $\exp \left(\lambda_{\infty} \int_{0}^{\infty}\left[\varphi_{x}^{o}(s)-1\right] d x\right)$ is the CF of some distribution $\nu$ on $\mathbb{R}_{+}^{k}$. To be more precise, we observe that, when $s \in(-1,1)^{k}$, the upper bound $\kappa$ in (29) can 
be chosen independently from $s \in(-1,1)^{k}$ as $\max _{j=1, \ldots, k} 1 / u_{j}$, a dominated convergence argument subsequently implies this continuity property.

Proving the limit of $e^{-\delta t} N(t)$ in (11) as $t \rightarrow+\infty$. We assume here that $\rho \leq 0$ and $\delta>0$ or that $\rho>0$ and $\delta>\rho$. First, the CF of $e^{-\delta t} N(t)$ is given as $e^{R_{t}}$ where $R_{t}$ can be written as below:

$$
\begin{aligned}
R_{t} & =\int_{0}^{t}\left[\varphi_{t-x}^{o}\left(s e^{-\delta t}\right)-1\right] \lambda(x) d x=\int_{0}^{t}\left\{\mathbb{E}\left[\exp \left(<i s e^{-\delta t}, N^{o}(t-x)>\right)\right]-1\right\} \lambda(x) d x(30) \\
& =R_{1, t}+R_{2, t}, \\
R_{1, t} & :=\int_{0}^{\infty} \mathbb{1}_{[0 \leq x \leq t]} \mathbb{E}\left[\exp \left(<i s e^{-\delta t}, N^{o}(t-x)>\right)-1-<i s e^{-\delta t}, N^{o}(t-x)>\right] \lambda(x) d x \\
R_{2, t} & :=\int_{0}^{\infty} \mathbb{1}_{[0 \leq x \leq t]} \mathbb{E}\left[<i s e^{-\delta t}, N^{o}(t-x)>\right] \lambda(x) d x
\end{aligned}
$$

Similar to (18), we then study the limits of $R_{1, t}$ and $R_{2, t}$ separately as $t \rightarrow+\infty$.

Limit of $R_{1, t}$ in (31) as $t \rightarrow+\infty$. It will be shown that $R_{1, t}$ tends to 0 as $t \rightarrow+\infty$. Let us first note that for all $x \in \mathbb{R}$ we have $\left|e^{i x}-1-i x\right| \leq|x|^{2}$ if $|x| \leq 1$, and (using $\left|e^{i x}-1\right| \leq|x|$ ) $\left|e^{i x}-1-i x\right| \leq\left|e^{i x}-1\right|+|i x| \leq 2|x| \leq 2|x|^{2}$ if $|x|>1$, so that we have the following general inequality

$$
\left|e^{i x}-1-i x\right| \leq 2|x|^{2}, \quad \forall x \in \mathbb{R} .
$$

The above result, combined with the Cauchy-Schwarz inequality, yields the upper bound for $\left|R_{1, t}\right|$ given by

$$
\begin{aligned}
\left|R_{1, t}\right| \leq 2 \int_{0}^{\infty} \mathbb{1}_{[0 \leq x \leq t]} \mathbb{E}\left[\mid<s e^{-\delta t}\right. & \left., N^{o}(t-x)>\left.\right|^{2}\right] \lambda(x) d x \\
& \leq 2\|s\|^{2} \int_{0}^{\infty} \mathbb{1}_{[0 \leq x \leq t]} e^{-2 \delta t} \mathbb{E}\left[\left\|N^{o}(t-x)\right\|^{2}\right] \lambda(x) d x .
\end{aligned}
$$

We here separate the cases $\rho<0, \rho=0$ and $\rho>0$, with last case requiring the additional constraint $\delta>\rho$. If $\rho<0$, the growth rate in Lemma 16 (with proof in Appendix A) implies that $\mathbb{E}\left[\left\|N^{o}(t-x)\right\|^{2}\right] \leq C e^{\rho(t-x)}$ for some constant $C>0$. Also, the assumption $\lambda(x) \sim$ $\lambda_{\infty} e^{\delta x}$ as $x \rightarrow \infty$, in particular implies that $\lambda(x)$ is bounded by $e^{\delta x}$ up to a constant, hence one gets from (33) that for some (different) constant $C>0$,

$$
\begin{aligned}
\left|R_{1, t}\right| \leq C \int_{0}^{\infty} \mathbb{1}_{[0 \leq x \leq t]} e^{-2 \delta t} e^{\rho(t-x)} e^{\delta x} d x & =C e^{(-2 \delta+\rho) t} \int_{0}^{t} e^{(-\rho+\delta) x} d x \\
& =\frac{C}{-\rho+\delta}\left[e^{-\delta t}-e^{(-2 \delta+\rho) t}\right] \longrightarrow 0 \text { as } t \rightarrow+\infty .
\end{aligned}
$$

If $\rho=0$, the growth rate in Lemma 16 implies that $\mathbb{E}\left[\left\|N^{o}(t-x)\right\|^{2}\right]$ is an $O((t-x)+1)$, hence for some constant $C>0$ we have

$$
\begin{aligned}
\left|R_{1, t}\right| \leq C \int_{0}^{\infty} \mathbb{1}_{[0 \leq x \leq t]} e^{-2 \delta t}[(t-x)+1] e^{\delta x} d x & \leq C(t+1) \int_{0}^{t} e^{-2 \delta t} e^{\delta x} d x \\
= & \frac{C(t+1)}{\delta}\left[e^{-\delta t}-e^{-2 \delta t}\right] \longrightarrow 0 \quad \text { as } t \rightarrow+\infty
\end{aligned}
$$


Finally, if $\rho>0$ then the growth rate in Lemma 16 implies that $\mathbb{E}\left[\left\|N^{o}(t-x)\right\|^{2}\right]$ is less than $e^{2 \rho(t-x)}$ up to a constant, hence for some constant $C>0$ we have

$$
\left|R_{1, t}\right| \leq C \int_{0}^{\infty} \mathbb{1}_{[0 \leq x \leq t]} e^{-2 \delta t} e^{2 \rho(t-x)} e^{\delta x} d x=C e^{2(-\delta+\rho) t} \int_{0}^{t} e^{(-2 \rho+\delta) x} d x
$$

which can be shown approaching 0 as $t \rightarrow+\infty$, using $\delta>\rho$.

Limit of $R_{2, t}$ in (32) as $t \rightarrow+\infty$, and conclusion. From [5, p.208], we know that the mean matrix of the multitype process $N^{o}(z)$ is expressed as $\mathbb{E}\left[N^{o}(z)\right]=e^{A z} \mathbb{E}(I)$ where the matrix $A$ is defined in (5). Therefore, $R_{2, t}$ can be expressed, after some manipulation, as

$$
\begin{aligned}
R_{2, t} & =\int_{0}^{t} e^{-\delta t}<i s, e^{A(t-x)} \mathbb{E}(I)>\lambda(x) d x \\
& =\int_{0}^{\infty} \mathbb{1}_{[0 \leq x \leq t]} e^{-\delta t}<i s, e^{A x} \mathbb{E}(I)>\lambda(t-x) d x
\end{aligned}
$$

We now wish to use the dominated convergence theorem in order to find the limit in (35). Upper bounding $\lambda(x)$ by $C e^{\delta x}$ for some constant $C>0$ results in

$$
\left|\mathbb{1}_{[0 \leq x \leq t]} e^{-\delta t}<i s, e^{A x} \mathbb{E}(I)>\lambda(t-x)\right| \leq C<|s|, e^{(A-\delta \mathbf{I d}) x} \mathbb{E}(I)>,
$$

which is integrable because $\delta>\rho$ (in either case $\rho \leq 0$ or $\rho>0$ ) so that all eigenvalues of $A-\delta$ Id have negative real parts. Also, the assumption that $\lambda(x) \sim \lambda_{\infty} e^{\delta x}$ as $x \rightarrow \infty$, results in $\mathbb{1}_{[0 \leq x \leq t]} e^{-\delta t}<i s, e^{A x} \mathbb{E}(I)>\lambda(t-x) \longrightarrow \lambda_{\infty}<i s, e^{(A-\delta \operatorname{Id}) x} \mathbb{E}(I)>$ as $t \rightarrow+\infty$, for all $x \geq 0$. Hence we deduce from (35) that

$$
\begin{aligned}
R_{2, t} \longrightarrow & \lambda_{\infty} \int_{0}^{\infty}<i s, e^{(A-\delta \mathrm{Id}) x} \mathbb{E}(I)>d x=<i s, \lambda_{\infty} \int_{0}^{\infty} e^{(A-\delta \mathrm{Id}) x} d x \mathbb{E}(I)> \\
= & <i s, \lambda_{\infty}(\delta \mathrm{Id}-A)^{-1} \mathbb{E}(I)>
\end{aligned}
$$

Since $R_{1, t} \longrightarrow 0$ as $t \rightarrow+\infty$, one arrives at the convergence of the $\mathrm{CF}$ of $e^{-\delta t} N(t)$ to $\exp (<$ $i s, \lambda_{\infty}(\delta \mathrm{Id}-A)^{-1} \mathbb{E}(I)>$ ), so that $e^{-\delta t} N(t)$ converges in distribution (or, equivalently, in probability) towards $\lambda_{\infty}(\delta \mathrm{Id}-A)^{-1} \mathbb{E}(I)$. Hence (ii) in (11) is proved.

Proving the limit of $e^{-\delta t} \frac{N(t)}{t}$ in (12) as $t \rightarrow+\infty$. We assume the supercritical case $\rho>0$ and $\rho=\delta$. In this case, instead of (30), we consider the quantity $R_{t}:=\int_{0}^{t}\left[\varphi_{t-x}^{o}\left(s e^{-\delta t} / t\right)-\right.$ $1] \lambda(x) d x=\int_{0}^{t}\left\{\mathbb{E}\left[\exp \left(<i s e^{-\delta t} / t, N^{o}(t-x)>\right)\right]-1\right\} \lambda(x) d x$ such that the CF of $e^{-\delta t} \frac{N(t)}{t}$ is equal to $e^{R_{t}}$, which is similarly decomposed as in (31) and (32) as $R_{t}=R_{1, t}+R_{2, t}$, where

$$
\begin{aligned}
R_{1, t} & :=\int_{0}^{\infty} \mathbb{1}_{[0 \leq x \leq t]} \mathbb{E}\left[\exp \left(<i s e^{-\delta t} / t, N^{o}(t-x)>\right)-1-<i s e^{-\delta t} / t, N^{o}(t-x)>\right] \lambda(x) d x, \\
R_{2, t} & :=\int_{0}^{\infty} \mathbb{1}_{[0 \leq x \leq t]} \mathbb{E}\left[<i s e^{-\delta t} / t, N^{o}(t-x)>\right] \lambda(x) d x .
\end{aligned}
$$

Utilizing the inequality in (34), one obtains, for some constant $C>0$,

$$
\left|R_{1, t}\right| \leq \frac{1}{t^{2}} C \int_{0}^{\infty} \mathbb{1}_{[0 \leq x \leq t]} e^{-2 \delta t} e^{2 \rho(t-x)} e^{\delta x} d x=C \frac{1}{t^{2}} \int_{0}^{t} e^{-\delta x} d x \longrightarrow 0
$$


thus (36) tends to 0 as $t \rightarrow+\infty$. Using the expression $\mathbb{E}\left[N^{o}(z)\right]=e^{A z} \mathbb{E}(I)$ as in (35) and the change of variable $z:=1-x / t$ together with the assumption $\delta=\rho$, we can rewrite (37) as

$$
\begin{aligned}
R_{2, t} & =\frac{1}{t} \int_{0}^{t} e^{-\delta t}<i s, e^{A(t-x)} \mathbb{E}(I)>\lambda(x) d x \\
& =<i s, \int_{0}^{1} e^{A t(1-z)} e^{-\rho t} \lambda(t z) d z \mathbb{E}(I)> \\
& =<i s, \int_{0}^{1} e^{(A-\rho \mathrm{Id}) t(1-z)} e^{-\rho t z} \lambda(t z) d z \mathbb{E}(I)>.
\end{aligned}
$$

We now wish to investigate (38) when $t \rightarrow+\infty$. Since $A$ is regular, the Perron Frobenius theory entails that $e^{(A-\rho \mathrm{Id}) x}$ converges to $u v^{\prime}$ as $x \rightarrow+\infty$, see e.g. [5, Limit (17) p.203]. Hence, for all $z \in(0,1)$ one has $\lim _{t \rightarrow \infty} e^{(A-\rho \operatorname{Id}) t(1-z)}=u v^{\prime}$. Also, the assumption $\lambda(x) \sim \lambda_{\infty} e^{\delta x}$ as $x \rightarrow \infty$ with $\delta=\rho$ implies that $\lim _{t \rightarrow \infty} e^{-\rho t z} \lambda(t z)=\lambda_{\infty}$ for all $z \in(0,1)$, so that by the dominated convergence theorem we may let $t \rightarrow+\infty$ in (38) and obtain

$$
R_{2, t} \longrightarrow<i s, \lambda_{\infty} u v^{\prime} \mathbb{E}(I)>, \quad t \rightarrow+\infty .
$$

Therefore, since $R_{t}=R_{1, t}+R_{2, t}$ with $\lim _{t \rightarrow \infty} R_{1, t}=0$, one concludes the convergence (12).

\subsection{Proof of Theorem 5 in the critical case $\rho=0$}

We start with Lemma 2, from which we deduce that the CF of $N(t) / t$ admits the expression

$$
\mathbb{E}\left[\exp \left(<i s, \frac{N(t)}{t}>\right)\right]=\mathbb{E}\left[\exp \left(<t^{-1} i s, N(t)>\right)\right]=\exp \left\{\int_{0}^{t}\left[\varphi_{t-y}^{o}\left(t^{-1} s\right)-1\right] \lambda(y) d y\right\} .
$$

We thus study

$$
\begin{aligned}
\int_{0}^{t}\left[\varphi_{t-y}\left(t^{-1} s\right)-1\right] \lambda(y) d y & =\int_{0}^{t} \mathbb{E}\left[\exp \left(<i s, \frac{N^{o}(t-y)}{t}>\right)-1\right] \lambda(y) d y \\
& =\int_{0}^{\Lambda(t)} \mathbb{E}\left[\exp \left(<i s, \frac{N^{o}\left(t-\Lambda^{-1}(y)\right)}{t}>\right)-1\right] d y \\
& =\int_{0}^{1} \Lambda(t) \mathbb{E}\left[\exp \left(<i s, \frac{N^{o}\left(t-\Lambda^{-1}(\Lambda(t) x)\right)}{t}>\right)-1\right] d x \\
& :=-\int_{0}^{1} \gamma_{t}(x) d x
\end{aligned}
$$

where $\Lambda^{-1}($.$) is the inverse of the function \Lambda($.) (invertible as it is assumed that $\lambda(t)>0$ for all $t \geq 0)$, the second last equality is due to a change of variable with $x:=y / \Lambda(t)$ and $\gamma_{t}(x)$ is given by

$$
\gamma_{t}(x):=\Lambda(t) \mathbb{E}\left[1-\exp \left(<i s, \frac{N^{o}\left(t-\Lambda^{-1}(\Lambda(t) x)\right)}{t}>\right)\right] .
$$

We note that the assumption $\lim _{t \rightarrow+\infty} \Lambda(t) / t=\lambda_{\infty}$ implies that $\lim _{t \rightarrow+\infty} \Lambda^{-1}(t) / t=\lambda_{\infty}^{-1}$, which is in turn equivalent to

$$
\Lambda^{-1}(t) \sim \lambda_{\infty}^{-1} t, \quad \text { i.e. } \quad \Lambda^{-1}(t)=\lambda_{\infty}^{-1} t+\eta(t) t,
$$


where $\lim _{t \rightarrow \infty} \eta(t)=0$.

In the following, we shall prove by the dominated convergence theorem that the right-hand side of (40) has the following limit

$$
-\int_{0}^{1} \gamma_{t}(x) d x \longrightarrow \lambda_{\infty} \bar{\beta} \int_{0}^{\infty} \mathbb{E}\left[\exp \left(<i s, e^{-y} \mathcal{X}>\right)-1\right] d y, \quad t \rightarrow+\infty
$$

where $\bar{\beta}$ is given by (14). In here we have

$$
\mathcal{X}=\chi v \otimes \mu^{-1} \in[0,+\infty)^{k},
$$

where $\chi \sim \mathcal{E}(c)$ for $c>0$ given by (15) and the survival function of $\mathcal{X}$ given by

$$
\mathbb{P}(\mathcal{X}>z)=\exp \left(-c \max _{i=1, \ldots, k} \frac{z_{i}}{v_{i} \mu_{i}^{-1}}\right), \quad z=\left(z_{1}, \ldots, z_{k}\right) \in[0,+\infty)^{k} .
$$

The proof is decomposed in the following steps.

Step 1: Dominating the integrand in (40). Using the basic inequality $\left|e^{i x}-1\right| \leq x$, we have for all $t \geq 0$ and $x \in(0,1), s=\left(s_{1}, \ldots, s_{k}\right) \in \mathbb{R}^{k}$ that

$$
\left|1-\exp \left(<i s, \frac{N^{o}\left(t-\Lambda^{-1}(\Lambda(t) x)\right)}{t}>\right)\right| \leq<|s|, \frac{N^{o}\left(t-\Lambda^{-1}(\Lambda(t) x)\right)}{t}>.
$$

Using (24), one finds $<|s|, \frac{N^{o}\left(t-\Lambda^{-1}(\Lambda(t) x)\right)}{t}>\leq \kappa\left(<u, \frac{N^{o}\left(t-\Lambda^{-1}(\Lambda(t) x)\right)}{t}>\right)$. Hence, taking the expectation and multiplying by $\Lambda(t)$ on both sides result in

$$
\begin{aligned}
& \left|\gamma_{t}(x)\right| \leq \Lambda(t) \kappa \mathbb{E}\left[<u, \frac{N^{o}\left(t-\Lambda^{-1}(\Lambda(t) x)\right)}{t}>\right] \leq C_{\lambda} \kappa \mathbb{E}\left[<u, N^{o}\left(t-\Lambda^{-1}(\Lambda(t) x)\right)>\right] \\
= & C_{\lambda} \kappa \mathbb{E}\left[<u, N^{o}(0)>\right]=C_{\lambda} \kappa<u, \mathbb{E}(I)>, \quad t \geq t_{0},
\end{aligned}
$$

where the first equality is obtained by the martingale argument and $C_{\lambda}:=\sup _{t \geq t_{0}} \Lambda(t) / t$ which is finite for a large enough $t_{0}$. Since $C_{\lambda}$ and $\kappa$ are constants (independent of $t$ and $x$ ), the integrand in (40) is dominated by some constant independent from $t \geq t_{0}$ and $x \in(0,1)$.

Step 2: Almost sure limit of the integrand in (40). Let us now prove the following convergence for $(41)$ :

$$
\gamma_{t}(x) \longrightarrow \gamma(x):=\lambda_{\infty} \frac{\bar{\beta}}{1-x} \mathbb{E}[1-\exp (<i s,(1-x) \mathcal{X}>)],
$$

for a fixed $x \in(0,1)$ and $s=\left(s_{1}, \ldots, s_{k}\right) \in \mathbb{R}^{k}$ as $t \rightarrow+\infty$, where $\bar{\beta}$ is defined by (14). To show this, we first express $\gamma_{t}(x)$ in (41) using the representation (3) as

$$
\begin{aligned}
\gamma_{t}(x) & =\sum_{\mathbf{n}_{0}=\left(\mathbf{n}_{0}(1), \ldots, \mathbf{n}_{0}(k)\right) \in \mathbb{N}^{k}} \gamma_{t}\left(x, \mathbf{n}_{0}\right) p_{\mathbf{n}_{0}} \\
\text { where } \gamma_{t}\left(x, \mathbf{n}_{0}\right) & :=\Lambda(t)\left[1-\mathbb{E}\left(\exp \left(<i s, \frac{N^{o}\left(t-\Lambda^{-1}(\Lambda(t) x)\right)}{t}>\right) \mid N^{o}(0)=\mathbf{n}_{0}\right)\right] \\
& =\Lambda(t)\left[1-\prod_{j=1}^{k} \psi^{j}(t, x)^{\mathbf{n}_{0}(j)}\right]
\end{aligned}
$$




$$
\psi^{j}(t, x):=\mathbb{E}\left[\exp \left(<i s, \frac{N^{j}\left(t-\Lambda^{-1}(\Lambda(t) x)\right)}{t}>\right)\right], \quad j=1, \ldots, k .
$$

In the following, we define for all $J \subset\{1, \ldots, k\}$ and $z=\left(z_{1}, \ldots, z_{k}\right) \in \mathbb{R}_{+}^{* k}$ the vector $z^{J}$ of which the $j$ th entry $z_{j}^{J}$ is $z_{j}$ if $j \in J$, and some arbitrary negative value (e.g. -1 ) otherwise. The following lemma, proved in Appendix B, gives the asymptotic behaviour of $\psi^{j}(t, x)$, $j=1, \ldots, k$, as $t \rightarrow \infty$, which helps us prove (47).

Lemma 8. The following limit holds for all $j=1, \ldots, k$ and $x \in(0,1)$ :

$$
t\left[\psi^{j}(t, x)-1\right] \longrightarrow \frac{1}{1-x} \beta_{j}[1-\mathbb{E}(\exp [<i s,(1-x) \mathcal{X}>])], \quad t \rightarrow \infty .
$$

Note that (51) in particular implies that $\psi^{j}(t, x)-1=O(1 / t) \longrightarrow 0$ as $t \rightarrow \infty$, which, plugged into (49) together with $\Lambda(t) \sim \lambda_{\infty} t$ as $t \rightarrow+\infty$, yields

$$
\gamma_{t}\left(x, \mathbf{n}_{0}\right) \sim \lambda_{\infty}\left[\sum_{j=1}^{k} \mathbf{n}_{0}(j) t\left[\psi^{j}(t, x)-1\right]\right], \quad t \rightarrow+\infty
$$

This implies, along with (51), that (48) converges as

$$
\gamma_{t}(x) \longrightarrow \frac{\lambda_{\infty}}{1-x}\left\{\sum_{j=1}^{k} \beta_{j}\left[\sum_{\mathbf{n}_{0} \in \mathbb{N}^{k}} \mathbf{n}_{0}(j) p_{\mathbf{n}_{0}}\right]\right\}[1-\mathbb{E}(\exp [<i s,(1-x) \mathcal{X}>])], \quad t \rightarrow+\infty
$$

and thus (47) holds.

Step 3: Proof of (43). Thanks to (46) and (47), by the dominated convergence theorem, one thus deduces that (40) converges as $t \rightarrow+\infty$ to

$$
-\int_{0}^{1} \gamma(x) d x=-\int_{0}^{1} \frac{\lambda_{\infty} \bar{\beta}}{1-x} \mathbb{E}[1-\exp (<i s,(1-x) \mathcal{X}>)] d x
$$

which results in (43) after a change of variable $y:=-\ln (1-x)$.

Step 4: End of proof. From (39) with the convergence results of (40) towards (43), one finds that

$$
\mathbb{E}\left[\exp \left(<i s, \frac{N(t)}{t}>\right)\right] \longrightarrow \exp \left(\lambda_{\infty} \bar{\beta} \int_{0}^{\infty} \mathbb{E}\left[\exp \left(<i s, e^{-y} \mathcal{X}>\right)-1\right] d y\right), \quad t \rightarrow+\infty
$$

for $s \in \mathbb{R}^{k}$. Since $\mathcal{X}=\chi v \otimes \mu^{-1}$ with $\chi \sim \mathcal{E}(c)$, one computes that $\mathbb{E}\left[\exp \left(<i s, e^{-t} \mathcal{X}>\right.\right.$ )$-1]=\mathbb{E}\left[\exp \left(<i s, v \otimes \mu^{-1}>\chi e^{-t}\right)-1\right]=\frac{e^{-t}<i s, v \otimes \mu^{-1}>}{c-e^{-t}<i s, v \otimes \mu^{-1}>}$. In turn, changing of variable $z:=e^{-t}\left\langle i s, v \otimes \mu^{-1}>\right.$ yields that the right-hand side of the above convergence is the CF equivalent to $\left(\frac{c}{c-<i s, v \otimes \mu^{-1}>}\right)^{\lambda_{\infty} \bar{\beta}}$, which indeed is the CF of $\mathcal{Z} v \otimes \mu^{-1}$ in (16). This completes the proof.

\section{Immigration modelled by a contagious Poisson process (CPP)}

As discussed in Section 1, CPP is a special case of GPP which has become a well-known contagion model when the transition intensity in the non-homogeneous birth process is a linear 
function of the current state multiplied by a function of the current time. In this section, we now assume that the arrival process $\{S(t), t \geq 0\}$ is a CPP and note that $S\left(0^{-}\right)=0$. We first consider GPP and obtain the CF of $N(t)$. This will in turn imply that the one for a CPP is available. It is known that GPP (or a positive contagion model in $[8,35]$ ), is a particular case of self exciting counting process with intensity rate $\lambda(t)$ satisfying

$$
\lambda(t)=\left[a S\left(t^{-}\right)+b\right] \lambda_{t}, \quad a>0, b>0,
$$

for some underlying function $\lambda_{t}>0$ for $t \in(0,+\infty)$ which is assumed to be continuous and integrable over finite ranges. Let us denote $\Lambda_{t}=\int_{0}^{t} \lambda_{y} d y$ for $t \geq 0$. When $b=1$, this arrival process was referred to as the Linear Extension of the Yule Process by [23]. Hence, the intensity increases linearly with the number of arrivals at time $t$. This explains why these models could be appropriate for the situations where the arriving particles representing cells infected by a rapidly expanding disease contaminate other cells in an organism modelled by a certain network mechanism, or where the occurrence of shocks causes outages of interconnected lines in a power system as studied in [26]. In particular, we shall focus on the case when $\lambda_{t}=\lambda>0$ is constant. In this case, $\{S(t), t \geq 0\}$ is called a CPP in [1] and [32].

Let us start by establishing the CF of $N(t)$ as obtained in Lemma 2 for the NHPP immigration.

Lemma 9. When the new particle arrives according to a GPP with the intensity rate given in (53), the CF of $N(t)$ in (7) admits the following expression

$$
\varphi_{t}(s)=\left\{1-\int_{0}^{t}\left[\varphi_{t-y}^{o}(s)-1\right] a \lambda_{y} e^{a \Lambda_{y}} d y\right\}^{-b / a} .
$$

In particular, when $\lambda_{t}=\lambda$ in (53) (i.e. CPP case), (54) is simplified as

$$
\varphi_{t}(s)=\left\{1-\int_{0}^{t}\left[\varphi_{t-y}^{o}(s)-1\right] a \lambda e^{a \lambda y} d y\right\}^{-b / a} .
$$

Proof. It is known that the marginal distribution of $S(t)$ is expressed as a negative binomial distribution with $\Lambda_{t}=\int_{0}^{t} \lambda_{y} d y$ (e.g. [9, Theorem 1(i)]) given by

$$
p_{t}(n):=\mathbb{P}(S(t)=n)=\frac{\Gamma(b / a+n)}{\Gamma(b / a) n !}\left(1-e^{-a \Lambda_{t}}\right)^{n}\left(e^{-a \Lambda_{t}}\right)^{b / a},
$$

where $\Gamma(z)=\int_{0}^{\infty} x^{z-1} e^{-x} d x$, for all complex number $z$ with positive real part, is the gamma function. The above is a negative binomial distribution $(r, p)$ where $r=b / a$ and $p=1-e^{-a \Lambda_{t}}$. Its probability generating function is $P_{t}(z)=\sum_{n=0}^{\infty} z^{n} p_{t}(n)=\left(\frac{1-p}{1-p z}\right)^{r}$ for all complex number $z$ verifying $|z|<p^{-1}$.

Then, from [21, Section 3.2], the CF of $N(t)$ can be expressed as a compound negative binomial distribution

$$
\varphi_{t}(s)=P_{t}\left(\widetilde{f}_{t}(s)\right)
$$

where the CF of the secondary distribution is given by

$$
\widetilde{f}_{t}(s)=\int_{0}^{t} q_{t}(y) \varphi_{t-y}^{o}(s) d y,
$$


with

$$
q_{t}(y)=\frac{a \lambda_{y} e^{a \Lambda_{y}}}{e^{a \Lambda_{t}}-1}, \quad 0 \leq y \leq t
$$

Since $P_{t}(z)=\left(\frac{1-p}{1-p z}\right)^{r},(56)$ is obtained as

$$
\varphi_{t}(s)=\left(\frac{e^{-a \Lambda_{t}}}{1-\left(1-e^{-a \Lambda_{t}}\right) \widetilde{f}_{t}(s)}\right)^{b / a}=\left[e^{a \Lambda_{t}}-\left(e^{a \Lambda_{t}}-1\right) \widetilde{f}_{t}(s)\right]^{-b / a} .
$$

Using $\int_{0}^{t} a \lambda_{y} e^{a \Lambda_{y}} d y=e^{a \Lambda_{t}}-1$, one finds from (57) and (58) that

$$
\begin{aligned}
e^{a \Lambda_{t}}-\left(e^{a \Lambda_{t}}-1\right) \widetilde{f}_{t}(s) & =\int_{0}^{t} a \lambda_{y} e^{a \Lambda_{y}} d y+1-\int_{0}^{t} a \lambda_{y} e^{a \Lambda_{y}} \varphi_{t-y}^{o}(s) d y \\
& =1+\int_{0}^{t}\left[1-\varphi_{t-y}^{o}(s)\right] a \lambda_{y} e^{a \Lambda_{y}} d y
\end{aligned}
$$

That is,

$$
\varphi_{t}(s)=\left\{1+\int_{0}^{t}\left[1-\varphi_{t-y}^{o}(s)\right] a \lambda_{y} e^{a \Lambda_{y}} d y\right\}^{-b / a}
$$

or equivalently (54).

In the case of a constant baseline intensity $\lambda_{t}=\lambda$, taking the expectation on both sides of (53) yields $\mathbb{E}[\lambda(t)]=a \lambda \mathbb{E}\left[S\left(t^{-}\right)\right]+\lambda b$. Since $\mathbb{E}\left[S\left(t^{-}\right)\right]=\mathbb{E}[S(t)]$ and $\left\{S(t)-\int_{0}^{t} \lambda(s) d s, t \geq 0\right\}$ is a martingale (see e.g. [18, Proposition 8.3.2.1 p.467]), we arrive at $\mathbb{E}[\lambda(t)]=a \lambda \int_{0}^{t} \mathbb{E}[\lambda(s)] d s+\lambda b$ for all $t \geq 0$, from which the expected intensity has the closed form

$$
\mathbb{E}[\lambda(t)]=b \lambda e^{a \lambda t}, \quad t \geq 0 .
$$

We note that there is some resemblance between this exponential expression in (60) in the GPP case and the exponential asymptotic form $\lambda(t) \sim \lambda_{\infty} e^{\delta t}$ of the (deterministic) intensity appeared in Theorems 3 and 4 in the NHPP case. However, due to the randomness in time feature of the intensity in this case, we can observe different limiting behaviors of the branching process $N(t)$ with the CPP immigration. More precisely, in the following it is shown that the distributional behaviour changes depending on whether the largest eigenvalue $\rho$ of $A$ is less than, larger than, or equal to $a \lambda$. The main result of this section is given in the following theorem.

Theorem 10. Suppose that $\lambda_{t}=\lambda, a$ is defined by (53) and $\rho$ is the largest eigenvalue of the matrix $A$ with elements $a_{i j}$ defined by (5).

(1) If $\rho>a \lambda$, then

$$
e^{-\rho t} N(t) \stackrel{\mathcal{D}}{\longrightarrow} \mathcal{Z}_{T} v, \quad t \rightarrow+\infty,
$$

where $v$ is the left eigenvector of the matrix $A, T \sim \Gamma(b / a, 1)$ and $\left\{\mathcal{Z}_{t}, t \geq 0\right\}$ is an independent Lévy process with characteristic exponent $\psi(x):=\int_{\mathbb{R}}(1-\exp [i x z]) \Pi(d z), x \geq 0$. Here, $\Pi($. is defined by

$$
\Pi(d z):=\mathbb{E}\left[W^{a \lambda / \rho_{1}} \mathbb{1}_{[W \geq z]}\right] \frac{a \lambda}{\rho} z^{-a \lambda / \rho-1} \mathbb{1}_{[0<z<+\infty]} d z,
$$

and we recall that $W$ is characterized by its $\mathrm{CF}$ given by (6). 
(2) If $\rho<a \lambda$, then

$$
e^{-a \lambda t} N(t) \stackrel{\mathcal{D}}{\longrightarrow} \mathcal{Z} \gamma, \quad t \rightarrow+\infty,
$$

where $\mathcal{Z}$ is a $\mathrm{rv}$ distributed as $\Gamma(b / a, 1)$ and $\gamma$ is the vector defined by

$$
\gamma:=a \lambda(a \lambda \mathrm{Id}-A)^{-1} \mathbb{E}(I)
$$

(3) If $\rho=a \lambda$, then

$$
\frac{N(t)}{t} e^{-a \lambda t} \stackrel{\mathcal{D}}{\longrightarrow} \mathcal{Z} v, \quad t \rightarrow+\infty,
$$

where $\mathcal{Z}$ is a rv distributed as $\Gamma(b / a, \mathbb{E}[W] a \lambda)$.

Remark 11. In the case $\rho>a \lambda$ we may note that, since $\Pi($.$) defined by (62) has support$ on $(0,+\infty)$ and verifies $\int_{(0,+\infty)} \min (1, z) \Pi(d z)<+\infty$ (precisely because of the condition $\rho>a \lambda)$, the underlying Lévy process $\left\{\mathcal{Z}_{t}, t \geq 0\right\}$ in (61) belongs to the class of subordinators according to [20, Lemma 2.14, p.55].

Remark 12. As shown in (60), the expected intensity in the present CPP case has an exponential form, hence it is natural to compare the limiting convergence results in Theorem 10 to those in Theorems 3 and 4 in the NHPP case with asymptotic intensity $\lambda(t) \sim \lambda_{\infty} e^{\delta t}$ for $\delta>0$. With an analogy between $a \lambda$ and $\delta$, Table 1 summarizes the different directions of the supports of the limiting distributions obtained in (11), (12) and (9) for $\rho<\delta, \rho=\delta$ and $\rho>\delta$ in the NHPP case, to those in (63), (65) and (61) for $\rho<a \lambda, \rho=a \lambda$ and $\rho>a \lambda$ in the CPP case. Each value in Table 1, that all belong to $\mathbb{R}_{+}^{k}$, roughly shows the position in which the renormalized process $N(t)$ is located asymptotically in the corresponding case. Interestingly,

\begin{tabular}{|c|c|c|c|}
\hline & $\rho<\xi$ & $\rho=\xi$ & $\rho>\xi$ \\
\hline NHPP: $\xi=\delta$ & $(\xi \operatorname{Id}-A)^{-1} \mathbb{E}(I)$ & $u$ & $v$ \\
\hline CPP: $\xi=a \lambda$ & $(\xi \operatorname{Id}-A)^{-1} \mathbb{E}(I)$ & $v$ & $v$ \\
\hline
\end{tabular}

Direction of limiting distribution

the directions are the same except for $\rho=\delta$ in the NHPP case and $\rho=a \lambda$ in the CPP case, which are respectively given by the vectors $u$ and $v$.

The proofs of each case in Theorem 10 are provided in the following Section 4.1, Section 4.2 , and Section 4.3 respectively.

\subsection{Proof of Theorem 10 in the case $\rho>a \lambda$}

In (55), with the normalizing function $g(t)=e^{\rho t}$ we get

$$
\varphi_{t}(s / g(t))=\varphi_{t}\left(s e^{-\rho t}\right)=\left\{1+\int_{0}^{t}\left[1-\varphi_{t-y}^{o}\left(s e^{-\rho t}\right)\right] a \lambda e^{a \lambda y} d y\right\}^{-b / a}
$$

for all $s \in \mathbb{R}^{k}$. The proof is divided into two steps as follows.

Step 1: Studying the convergence of $\varphi_{t}\left(s e^{-\rho t}\right)$ as $t \rightarrow+\infty$. It is convenient to introduce the function

$$
\Xi_{t, s}:=\int_{0}^{t}\left[1-\varphi_{t-y}^{o}\left(s e^{-\rho t}\right)\right] a \lambda e^{a \lambda y} d y
$$




$$
=\int_{0}^{\infty} \mathbb{1}_{[0<y<t]} \mathbb{E}\left[1-\exp \left(<i s, N^{o}(t-y) / e^{\rho(t-y)}>e^{-\rho y}\right)\right] a \lambda e^{a \lambda y} d y,
$$

so that $\varphi_{t}\left(s e^{-\rho t}\right)=\left\{1+\Xi_{t, s}\right\}^{-b / a}, t \geq 0$. Thus, studying the limit of $\varphi_{t}\left(s e^{-\rho t}\right)$ as $t \rightarrow$ $+\infty$ essentially requires finding $\lim _{t \rightarrow+\infty} \Xi_{t, s}$, which will be completed by the dominated convergence theorem. First note that for all $y \in(0,+\infty)$ one has that $N^{o}(t-y) / e^{\rho(t-y)} \longrightarrow$ $W v, t \rightarrow \infty$, a.s. from Lemma 1 . One has by the dominated convergence theorem for a fixed $y \in(0,+\infty)$ and that

$$
\mathbb{E}\left[1-\exp \left(<i s, N^{o}(t-y) / e^{\rho(t-y)}>e^{-\rho y}\right)\right] \longrightarrow \mathbb{E}\left[1-\exp \left(<i s, v>W e^{-\rho y}\right)\right], \quad t \rightarrow+\infty .
$$

Using (22), the integrand in (67) is upper bounded in modulus as

$$
\begin{aligned}
& \mathbb{1}_{[0<y<t]}\left|\mathbb{E}\left[1-\exp \left(<i s, N^{o}(t-y) / e^{\rho(t-y)}>e^{-\rho y}\right)\right] a \lambda e^{a \lambda y}\right| \\
& \leq \mathbb{1}_{[0<y<t]} \mathbb{E}\left[<|s|, N^{o}(t-y) / e^{\rho(t-y)}>e^{-\rho y}\right] a \lambda e^{a \lambda y} \\
& =a \lambda \mathbb{1}_{[0<y<t]} \mathbb{E}\left[<|s|, N^{o}(t-y) / e^{\rho(t-y)}>\right] e^{(a \lambda-\rho) y}
\end{aligned}
$$

By the similar martingale argument applied to the one leading to (29) for example, and since Assumption (A) holds, one can show that $\left.\mathbb{1}_{[0<y<t]} \mathbb{E}\left[<|s|, N^{o}(t-y) / e^{\rho(t-y)}\right\rangle\right]$ is upper bounded by some constant $K$ which is independent of $t$ and $y$. That is,

$$
0 \leq \mathbb{1}_{[0<y<t]}\left|\mathbb{E}\left[1-\exp \left(<i s, N^{o}(t-y) / e^{\rho(t-y)}>e^{-\rho y}\right)\right]\right| a \lambda e^{a \lambda y} \leq a \lambda K e^{(a \lambda-\rho) y},
$$

which is integrable over $y \in(0,+\infty)$ when $\rho>a \lambda$. Hence, using (68), (69) and the dominated convergence theorem, we arrive at

$$
\Xi_{t, s} \longrightarrow \Xi_{\infty, s}:=\int_{0}^{\infty} \mathbb{E}\left[1-\exp \left(<i s, v>W e^{-\rho y}\right)\right] a \lambda e^{a \lambda y} d y, \quad t \rightarrow+\infty
$$

so that the renormalized $\mathrm{CF}$ in (66) converges as

$$
\varphi_{t}\left(s e^{-\rho t}\right) \longrightarrow \tilde{\varphi}(s):=\left\{1+\Xi_{\infty, s}\right\}^{-b / a}, \quad t \rightarrow+\infty .
$$

Step 2: Identifying the $\mathbf{C F} \tilde{\varphi}(s)$. In order to interpret (71) as the convergence towards some known distribution, we use the following elementary Lemma (its proof is given in Appendix C):

Lemma 13. Let $\left\{\mathcal{Z}_{t}, t \geq 0\right\}$ be a Lévy process with characteristic exponent $\psi(x)$ such that $\mathbb{E}\left[e^{i x \mathcal{Z}_{t}}\right]=e^{-t \psi(x)}$ for $x \in \mathbb{R}$, and let $T$ be a $\mathrm{rv}$ distributed as $\Gamma(\zeta, 1)$, independent from $\left\{\mathcal{Z}_{t}, t \geq 0\right\}$. Then the $\mathrm{CF}$ of $\mathcal{Z}_{T}$ is given by

$$
\mathbb{E}\left[e^{i x \mathcal{Z}_{T}}\right]=\{1+\psi(x)\}^{-\zeta}, \quad x \geq 0 .
$$

The aim is now to write $\tilde{\varphi}(s)$ in (71) in the form of (72). We first write $\Xi_{\infty, s}$ in (70) as

$$
\Xi_{\infty, s}=\int_{0}^{\infty} \int_{0}^{\infty}\left(1-\exp \left[<i s, v>w e^{-\rho y}\right]\right) a \lambda e^{a \lambda y} d y \mathbb{P}(W \in d w) .
$$


Performing a change of variable $z:=w e^{-\rho y}$ (i.e. $y=-\frac{1}{\rho} \ln \frac{z}{w}$ ) within the integral in $y$, it may be expressed as

$$
\begin{aligned}
& \Xi_{\infty, s}=\int_{0}^{\infty} \int_{0}^{w}(1-\exp [<i s, v>z]) \frac{a \lambda}{\rho}\left(\frac{z}{w}\right)^{-a \lambda / \rho} \frac{d z}{z} \mathbb{P}(W \in d w) \\
& =\int_{0}^{\infty}(1-\exp [<i s, v>z])\left\{\int_{0}^{\infty} w^{a \lambda / \rho_{1}} \mathbb{1}_{[w \geq z]} \mathbb{P}(W \in d w)\right\} \frac{a \lambda}{\rho} z^{-a \lambda / \rho-1} d z \\
& =\int_{0}^{\infty}(1-\exp [<i s, v>z]) \mathbb{E}\left[W^{a \lambda / \rho_{1}}[W \geq z]\right] \frac{a \lambda}{\rho} z^{-a \lambda / \rho-1} d z \\
& =\int_{\mathbb{R}}(1-\exp [<i s, v>z]) \Pi(d z),
\end{aligned}
$$

where the measure $\Pi(d z)$ on $(0,+\infty)$ is defined as $(62)$. Finally, we get the following expression for $(71)$ :

$$
\tilde{\varphi}(s)=\{1+\psi(<s, v>)\}^{-b / a}, \quad s \in \mathbb{R}^{k},
$$

so that one deduces from Lemma 13 the convergence result in (61).

\subsection{Proof of Theorem 10 in the case $\rho<a \lambda$}

After a change of variable $y:=t-y,(55)$ is rewritten as

$$
\varphi_{t}(s)=\left\{1+\int_{0}^{t}\left[1-\varphi_{y}^{o}(s)\right] a \lambda e^{a \lambda(t-y)} d y\right\}^{-b / a}, \quad t \geq 0, \quad s \in \mathbb{R}^{k} .
$$

Let us consider the normalizing function $g(t)=e^{a \lambda t}$, so that

$$
\varphi_{t}(s / g(t))=\varphi_{t}\left(s e^{-a \lambda t}\right)=\left\{1+\int_{0}^{t}\left[1-\varphi_{y}^{o}\left(s e^{-a \lambda t}\right)\right] a \lambda e^{a \lambda(t-y)} d y\right\}^{-b / a} .
$$

In the following, the limit of the integral on the right-hand side of (73) is studied in the subcritical case. First, similar to (67), let

$$
\Xi_{t, s}:=\int_{0}^{t}\left[1-\varphi_{y}^{o}\left(s e^{-a \lambda t}\right)\right] a \lambda e^{a \lambda(t-y)} d y .
$$

To apply the dominated convergence theorem, let us define

$$
\Xi_{t, s, y}:=\mathbb{1}_{[0<y<t]}\left[1-\varphi_{y}^{o}\left(s e^{-a \lambda t}\right)\right] a \lambda e^{a \lambda(t-y)}=\mathbb{1}_{[0<y<t]} \mathbb{E}\left[1-e^{<i s, N^{o}(y)>e^{-a \lambda t}}\right] a \lambda e^{a \lambda(t-y)} .
$$

Since

$$
\left|1-e^{<i s, N^{o}(y)>e^{-a \lambda t}}\right| \leq<|s|, N^{o}(y)>e^{-a \lambda t},
$$

(75) is bounded in modulus by

$$
\begin{aligned}
\left|\Xi_{t, s, y}\right| & \leq \mathbb{1}_{[0<y<t]} \mathbb{E}\left[<|s|, N^{o}(y)>\right] a \lambda e^{-a \lambda y} \leq \mathbb{E}\left[<|s|, N^{o}(y)>\right] a \lambda e^{-a \lambda y} \\
& =<|s|, \mathbb{E}\left[N^{o}(y)\right]>a \lambda e^{-a \lambda y}:=\Xi_{s, y}^{*} .
\end{aligned}
$$

We recall from [5, p.202] that the mean matrix of the multitype process $N^{o}(t)$ is expressed as $\mathbb{E}\left[N^{o}(y)\right]=e^{A y} \mathbb{E}(I)$ where the matrix $A$ is defined in (5). For the case $\rho<a \lambda$, the integral 
$\int_{0}^{+\infty} e^{(A-a \lambda \mathrm{Id}) y} d y$ is convergent because all eigenvalues of the matrix $A-a \lambda \mathrm{Id}$ have negative real part in the case of $\rho<a \lambda$. In turn, one concludes that $\int_{0}^{+\infty} \Xi_{s, y}^{*} d y$ converges. Besides, for a fixed $y \in(0,+\infty)$ one finds

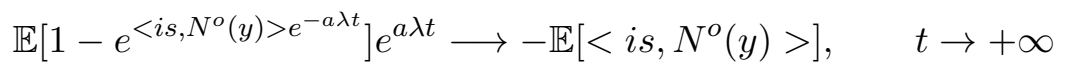

by the dominated convergence theorem. Indeed, from (76) $\left|1-e^{<i s, N^{o}(y)>e^{-a \lambda t}}\right| e^{a \lambda t}$ is upper bounded by $<|s|, N^{o}(y)>$ which has a finite expectation. Finally, because of the bound for the integrand $\Xi_{t, s, y}$ obtained in (77) and the pointwise limit in (78), one deduces that (74) converges to

$$
\begin{aligned}
\Xi_{t, s} \longrightarrow-\int_{0}^{+\infty} \mathbb{E}\left(<i s, N^{o}(y)>\right) a \lambda e^{-a \lambda y} d y & =-\int_{0}^{+\infty}<i s, e^{A y} \mathbb{E}(I)>a \lambda e^{-a \lambda y} d y \\
& =-<i s, \int_{0}^{+\infty} a \lambda e^{(A-a \lambda \operatorname{Id}) y} d y \mathbb{E}(I)>=<i s, a \lambda(a \lambda \operatorname{Id}-A)^{-1} \mathbb{E}(I)>
\end{aligned}
$$

as $t \rightarrow+\infty$. Consequently, it follows that (73) converges to

$$
\varphi_{t}\left(s e^{-a \lambda t}\right) \longrightarrow\left\{1-<i s, a \lambda(a \lambda \operatorname{Id}-A)^{-1} \mathbb{E}(I)>\right\}^{-b / a}, \quad t \rightarrow+\infty,
$$

for all $s \in \mathbb{R}^{k}$, which entails (63) with the vector $\gamma$ defined as (64).

\subsection{Proof of Theorem 10 in the case $\rho=a \lambda$}

We consider here the renormalizing function $g(t):=t e^{\rho t}=t e^{a \lambda t}$. As in (66) and (67), after a change of variable $y:=y / t$ we have for all $s \in \mathbb{R}^{k}$,

$$
\begin{aligned}
\varphi_{t}(s / g(t)) & =\varphi_{t}\left(s e^{-a \lambda t} / t\right)=\left\{1+\int_{0}^{t}\left[1-\varphi_{t-y}^{o}\left(s e^{-a \lambda t} / t\right)\right] a \lambda e^{a \lambda y} d y\right\}^{-b / a} \\
& =\left\{1+\int_{0}^{t}\left(1-\mathbb{E}\left[\exp \left(<i s, N^{o}(t-y)>e^{-a \lambda t} / t\right)\right]\right) a \lambda e^{a \lambda y} d y\right\}^{-b / a} \\
& =\left\{1+\int_{0}^{1} t\left(1-\mathbb{E}\left[\exp \left(<i s, N^{o}(t(1-y))>e^{-a \lambda t} / t\right)\right]\right) a \lambda e^{a \lambda t y} d y\right\}^{-b / a} \\
& =\left\{1+\Xi_{t, s}\right\}^{-b / a},
\end{aligned}
$$

where $\Xi_{t, s}$ is now defined by

$$
\begin{aligned}
\Xi_{t, s} & :=\int_{0}^{1} t\left(1-\mathbb{E}\left[\exp \left(<i s, N^{o}(t(1-y))>e^{-a \lambda t} / t\right]\right) a \lambda e^{a \lambda t y} d y\right. \\
& =\int_{0}^{1} t\left(1-\mathbb{E}\left[\exp \left(<i s, W v>e^{-a \lambda t y} / t\right)\right]\right) a \lambda e^{a \lambda t y} d y \\
& +\int_{0}^{1} t\left(\mathbb{E}\left[\exp \left(<i s, W v>e^{-a \lambda t y} / t\right)\right]-\mathbb{E}\left[\exp \left(<i s, N^{o}(t(1-y))>e^{-a \lambda t} / t\right)\right]\right) a \lambda e^{a \lambda t y} d y \\
& :=\Xi_{t, s}^{1}+\Xi_{t, s}^{2} .
\end{aligned}
$$


In the following we shall determine the limits of $\Xi_{t, s}^{1}$ and $\Xi_{t, s}^{2}$ separately as $t \rightarrow+\infty$. For notational convenience, let $\Xi_{t, s}^{2}:=\int_{0}^{1} \Upsilon_{s}^{2}(t, y) d y$ where

$\Upsilon_{s}^{2}(t, y):=t\left(\mathbb{E}\left[\exp \left(<i s, W v>e^{-a \lambda t y} / t\right)\right]-\mathbb{E}\left[\exp \left(<i s, N^{o}(t(1-y))>e^{-a \lambda t} / t\right)\right]\right) a \lambda e^{a \lambda t y}$.

Step 1: Studying the convergence of $\Xi_{t, s}^{1}$ as $t \rightarrow+\infty$. It is readily obtainable that using the inequality (22), one has for all $t \geq 0$ and $y \in(0,1)$ that

$$
\begin{aligned}
& t\left|1-\exp \left(<i s, W v>e^{-a \lambda t y} / t\right)\right| a \lambda e^{a \lambda t y} \\
& \leq t<|s|, W v>\left(e^{-a \lambda t y} / t\right) a \lambda e^{a \lambda t y}=<|s|, W v>a \lambda,
\end{aligned}
$$

which is integrable, so that for a fixed $y \in(0,1)$ one has by the dominated convergence theorem that $t\left(1-\mathbb{E}\left[\exp \left(<i s, W v>e^{-a \lambda t y} / t\right)\right]\right) a \lambda e^{a \lambda t y} \longrightarrow-\mathbb{E}[<i s, W v>] a \lambda$ as $t \rightarrow$ $+\infty$. Likewise:

$$
t\left|1-\mathbb{E}\left[\exp \left(<i s, W v>e^{-a \lambda t y} / t\right)\right]\right| a \lambda e^{a \lambda t y} \leq \mathbb{E}[<|s|, W v>] a \lambda,
$$

is a constant, so that by the dominated convergence theorem one deduces that

$$
\lim _{t \rightarrow+\infty} \Xi_{t, s}^{1}=-\mathbb{E}[<i s, W v>] a \lambda=-<i s, \mathbb{E}[W] a \lambda v>
$$

Step 2: Dominating $\Upsilon_{s}^{2}(t, y)$. In order to study $\lim _{t \rightarrow+\infty} \Xi_{t, s}^{2}$, we again use the dominated convergence theorem. First, it can be shown that $\left|\Upsilon_{s}^{2}(t, y)\right|$ in (81) is upper bounded as:

$$
\begin{aligned}
\left|\Upsilon_{s}^{2}(t, y)\right| & \leq t \mathbb{E}\left[\left|<s, W v>e^{-a \lambda t y} / t-<s, N^{o}(t(1-y))>e^{-a \lambda t} / t\right|\right] a \lambda e^{a \lambda t y} \\
& =a \lambda \mathbb{E}\left[\left(\left|<s, W v>-<s, N^{o}(t(1-y))>e^{-a \lambda t(1-y)}\right|\right]\right. \\
& \leq a \lambda \mathbb{E}[|<s, W v>|]+a \lambda \mathbb{E}\left[\left|<s, N^{o}(t(1-y))>e^{-a \lambda t(1-y)}\right|\right] \\
& \leq a \lambda \mathbb{E}[<|s|, W v>]+a \lambda \mathbb{E}\left[<|s|, N^{o}(t(1-y))>e^{-a \lambda t(1-y)}\right],
\end{aligned}
$$

where the first inequality is obtained from (22) and the last equality holds because $W$ and $N^{o}(t(1-y))$ are non negative or have non negative entries. Using again the constant $\kappa$ satisfying (24) and the martingale argument one thus obtains, together with the above result, that $\left|\Upsilon_{s}^{2}(t, y)\right|$ is upper bounded by some constant as

$$
\left|\Upsilon_{s}^{2}(t, y)\right| \leq a \lambda \mathbb{E}[<|s|, W v>]+a \lambda \kappa<u, \mathbb{E}(I)>, \quad \forall t \geq 0, \forall y \in(0,1) .
$$

Step 3: Pointwise convergence of $\Upsilon_{s}^{2}(t, y)$ towards 0 as $t \rightarrow+\infty$. Let $y \in(0,1)$ be fixed. Since $\mathbb{R}^{k}$ can be decomposed as the direct sum of $\mathbb{R} u$ and $(\mathbb{R} v)^{\perp}$ (the orthogonal vector space of $\mathbb{R} v$ for the euclidean inner product), there exists some (unique) $\alpha \in \mathbb{R}$ and $s_{0} \in(\mathbb{R} v)^{\perp}$ such that $s=\alpha u+s_{0}$. Since $\left\langle s_{0}, v\right\rangle=0$, it follows that (83) is expressed as

$$
\begin{aligned}
& \left|\Upsilon_{s}^{2}(t, y)\right| \leq a \lambda \mathbb{E}\left[\left|<s, W v>-<s, N^{o}(t(1-y))>e^{-a \lambda t(1-y)}\right|\right) \\
= & a \lambda \mathbb{E}\left(\left|\alpha<u, W v>-\alpha<u, N^{o}(t(1-y))>e^{-a \lambda t(1-y)}-<s_{0}, N^{o}(t(1-y))>e^{-a \lambda t(1-y)}\right|\right) \\
\leq & a \lambda \mathbb{E}\left(\left|\alpha W-\alpha<u, N^{o}(t(1-y))>e^{-a \lambda t(1-y)}\right|\right) \\
& +\mathbb{E}\left(\left|<s_{0}, N^{o}(t(1-y))>e^{-a \lambda t(1-y)}\right|\right)
\end{aligned}
$$

where the last line follows from the triangle inequality and the fact that $\langle u, W v\rangle=W<$ $u, v>=W .1=W$. We next show that both terms in the above inequality tend to zero as 
$t \rightarrow+\infty$. From this decomposition of $s$ along $\mathbb{R} u$ and $(\mathbb{R} v)^{\perp}$, it holds that the first term is linked to the martingale $\left\{\left\langle u, N^{o}(t) e^{-\rho t}\right\rangle, t \geq 0\right\}$ with $\rho=a \lambda$, whereas in the second term the behaviour of $\left\{\left\langle s_{0}, N^{o}(t) e^{-\rho t}\right\rangle, t \geq 0\right\}$ is determined precisely thanks to the estimates given in [4] and the fact that $s_{0} \in(\mathbb{R} v)^{\perp}$. Indeed, one has from (94) that $D(t)=O\left(e^{2 \rho t}\right)$, resulting in $\left(\mathbb{E}\left[\left\|N^{o}(t)\right\|^{2} e^{-2 \rho t}\right]\right)_{t \geq 0}$ is uniformly upper bounded with $\rho=a \lambda$ here. Since $\mathbb{E}[\mid<$ $\left.u, N^{o}(t)>\left.e^{-a \lambda t}\right|^{2}\right]$ is upper bounded by $\mathbb{E}\left[\left\|N^{o}(t)\right\|^{2} e^{-2 \rho t}\right]$ up to a constant for all $t \geq 0$, one deduces that the martingale $\left\{\left\langle u, N^{o}(t) e^{-a \lambda t}\right\rangle, t \geq 0\right\}$ is uniformly square integrable, hence converges in mean square towards $W$ as $t \rightarrow+\infty$; and in turn, the first term on the right-hand side of (84) converges to 0 as $t \rightarrow+\infty$. Concerning the second term, we have, using the representation (3) and triangular inequality, that

$$
\begin{aligned}
& \quad \sum_{\mathbf{n}_{0}=\left(\mathbf{n}_{0}(1), \ldots, \mathbf{n}_{0}(k)\right) \in \mathbb{N}^{k}} \mathbb{E}\left(\left|<s_{0}, N^{o}(t(1-y))>e^{-a \lambda t(1-y)}\right| \mid N^{o}(0)=\mathbf{n}_{0}\right) p_{\mathbf{n}_{0}} \\
& \leq \sum_{\mathbf{n}_{0}=\left(\mathbf{n}_{0}(1), \ldots, \mathbf{n}_{0}(k)\right) \in \mathbb{N}^{k}}\left\{\sum_{j=1}^{k} \mathbf{n}_{0}(j) \mathbb{E}\left(\left|<s_{0}, N^{j}(t(1-y))>e^{-a \lambda t(1-y)}\right|\right)\right\} p_{\mathbf{n}_{0}} \\
& \leq\left\{\sum_{\mathbf{n}_{0}=\left(\mathbf{n}_{0}(1), \ldots, \mathbf{n}_{0}(k)\right) \in \mathbb{N}^{k}}\left[\sum_{j=1}^{k} \mathbf{n}_{0}(j)\right] p_{\mathbf{n}_{0}}\right\} \max _{j=1, \ldots, k} \mathbb{E}\left(\left|<s_{0}, N^{j}(t(1-y))>e^{-a \lambda t(1-y)}\right|\right) \\
& =\left\{\sum_{j=1}^{k} \mathbb{E}(I(j))\right\} \max _{j=1, \ldots, k} \mathbb{E}\left(\left|<s_{0}, N^{j}(t(1-y))>e^{-a \lambda t(1-y)}\right|\right) \\
& \leq\left\{\sum_{j=1}^{k} \mathbb{E}(I(j))\right\} \max _{j=1, \ldots, k} \mathbb{E}\left(\left|<s_{0}, N^{j}(t(1-y))>\right|^{2} e^{-2 a \lambda t(1-y)} \mid\right)^{1 / 2}
\end{aligned}
$$

where the last line is obtained thanks to the Cauchy-Schwarz inequality. From [4, Proposition $3]$ together with $\left\langle s_{0}, v\right\rangle=0$, there exists some real number $a\left(s_{0}\right)<\rho=a \lambda$ as well as an integer $\gamma\left(s_{0}\right)$ (both depending on $s_{0}$, see their precise definitions in [4, (9a) and (9b)]) such that one of the three following situations occurs for all $j=1, \ldots, k$ :

$$
\mathbb{E}\left[\left|<s_{0}, N^{j}(t)>\right|^{2}\right]=\left\{\begin{array}{cl}
O\left(e^{2 a\left(s_{0}\right) t} t^{2 \gamma\left(s_{0}\right)}\right) & \text { if } 2 a\left(s_{0}\right)>\rho=a \lambda, \\
O\left(e^{2 a\left(s_{0}\right) t} t^{2 \gamma\left(s_{0}\right)+1}\right) & \text { if } 2 a\left(s_{0}\right)=\rho=a \lambda, \\
O\left(e^{\rho t}\right)=O\left(e^{a \lambda t}\right) & \text { if } 2 a\left(s_{0}\right)<\rho=a \lambda .
\end{array}\right.
$$

Here the above three cases are corresponding to [4, a), b) and c) of Proposition 3] respectively. In all cases, since $a\left(s_{0}\right)$ verifies $a\left(s_{0}\right)<\rho=a \lambda$, one can check easily that $\mathbb{E}\left[\mid<s_{0}, N^{j}(t)>\right.$ $\left.\left.\right|^{2}\right] e^{-2 \rho t}=\mathbb{E}\left[\left|<s_{0}, N^{j}(t)>\right|^{2}\right] e^{-2 a \lambda t}$ tends to 0 as $t \rightarrow+\infty$. Since Assumption (A) holds, (85) thus tends to 0 as $t \rightarrow+\infty$ (for a fixed $y \in(0,1)$ ). Combining all the above results, we thus prove that both terms on the right-hand side of (84) converge to 0 . Therefore, it is concluded that (81) goes to zero as $t \rightarrow+\infty$ for all $y \in(0,1)$.

Step 4: Convergence of $\Xi_{t, s}^{2}$ and conclusion. Step 2 and Step 3 imply by the dominated convergence theorem that $\lim _{t \rightarrow+\infty} \Xi_{t, s}^{2}=0$. Using this and (82), from (80) it follows that (79) converges to

$$
\varphi_{t}\left(s e^{-a \lambda t} / t\right) \longrightarrow\{1-<i s, \mathbb{E}[W] a \lambda v>\}^{-b / a}, \quad t \rightarrow+\infty .
$$

Hence we have proved (65). 


\section{Transient expectation when $k=2$}

We shall hereafter consider two-type branching processes (i.e. $k=2$ ) to study transient expectation of the number of particles at time $t$. Assume that the lifetime of type $j$ particles for $j=1,2$ is exponentially distributed as $\mathcal{E}\left(\mu_{i}\right)$. The branching mechanism is given by the following generating functions in (1)

$$
h_{1}\left(z_{1}, z_{2}\right)=p_{1}(0,0)+p_{1}(0,1) z_{2}, \quad h_{2}\left(z_{1}, z_{2}\right)=p_{2}(0,0)+p_{2}(1,0) z_{1}, \quad\left(z_{1}, z_{2}\right) \in[0,1]^{2},
$$

where probabilities $p_{12}:=p_{1}(0,1)$ and $p_{21}:=p_{2}(1,0)$ in $(0,1]$ satisfy $p_{12} p_{21}<1$, which means that type 1 particle (resp. 2) produces a type 2 (resp. 1) particle with probability $p_{12}$ (resp. $p_{21}$ ), or else dies with probability $p_{1}(0,0)=1-p_{12}$ (resp. $\left.p_{2}(0,0)=1-p_{21}\right)$. We also suppose that there is only one immigrant of type 1 entering the system at each time $T_{i}, i \in \mathbb{N}$, i.e. that $I \sim \delta_{(1,0)}$. We shall see in Remark 15 that this assumption can be relaxed to a general incoming immigration vector $I$ in (2). Also note that in this model, each particle produces one offspring only, and only one offspring of the other type. Finally, we denote by $m(t)=\mathbb{E}[S(t)]$ for $t \geq 0$ the renewal function associated to the immigration process $\{S(t), t \geq 0\}$ with the convention $m(t)=0$ when $t<0$.

Theorem 14. At time $t$, the transient expectation $\mathbb{E}\left[N_{1}(t)\right]$ for type 1 particle is given by

$$
\begin{aligned}
& \mathbb{E}\left[N_{1}(t)\right]=\int_{0}^{t}\left\{m(t)-m(t-z)+\int_{0}^{t}[m(t-v)-m(t-v-z)]\right. \\
&\left.\mu_{1} \mu_{2} p_{12} p_{21}\left[\frac{1}{\zeta_{1}\left(\zeta_{2}-\zeta_{1}\right)} e^{\zeta_{1} v}+\frac{1}{\zeta_{2}\left(\zeta_{1}-\zeta_{2}\right)} e^{\zeta_{2} v}\right] d v\right\} \mu_{1} e^{-\mu_{1} z} d z, \quad t \geq 0,
\end{aligned}
$$

where $\zeta_{1}$ and $\zeta_{2}$ are given by

$$
\begin{aligned}
\zeta_{1} & :=\frac{1}{2}\left[-\left(\mu_{1}+\mu_{2}\right)+\sqrt{\left(\mu_{1}-\mu_{2}\right)^{2}+4 \mu_{1} \mu_{2} p_{12} p_{21}}\right], \\
\zeta_{2} & :=\frac{1}{2}\left[-\left(\mu_{1}+\mu_{2}\right)-\sqrt{\left(\mu_{1}-\mu_{2}\right)^{2}+4 \mu_{1} \mu_{2} p_{12} p_{21}}\right] .
\end{aligned}
$$

Note that the expression (86) depends on the renewal function $m(t)$, which is explicitly available in many processes. For example, $m(t)=\int_{0}^{t} \lambda(s) d s$ when the immigration process is a NHPP with intensity $\lambda(\cdot)$ whereas $m(t)=\left(\frac{b}{a}\right) \frac{1-e^{-a \Lambda_{t}}}{e^{-a \Lambda_{t}}}$ when the immigration process is a GPP with parameters $\left(a, b, \lambda_{t}\right)$. In addition to these two processes considered in this paper, we remark that (86) for the transient first moment is also available for other non Poisson arrival processes where their renewal functions are known. Typical examples include the case when $\{S(t), t \geq 0\}$ is a fractional Poisson process with parameter $\beta \in(0,1)$ (where $m(t)=C t^{\beta}$ for some constant $C>0$, see [22, Expression (26)]), or when the interarrival times $T_{i}-T_{i-1}$, $i \geq 1$, follow matrix exponential distributions (in which case $m(t)$ is explicit and given by [3, Theorem 3.1]).

Proof. The key idea is to consider the successive passage times from type 2 to type 1 of the $i$ th particle arriving at $T_{i}, i \in \mathbb{N}^{*}$ which is type 1 . The type of particles is changing between 1 and 2 while it remains in the same type during an exponentially distributed lifetime as long as it is alive (i.e. it has not left the system). Let $G_{i}$ be the number of sojourn times as type 1 before dying, so that the $\left(G_{i}\right)_{i \in \mathbb{N}^{*}}$ are iid with distribution

$$
G_{i} \sim \mathcal{G}\left(1-p_{12} p_{21}\right)
$$


where $\mathcal{G}$ denotes the geometric distribution. In other words, $G_{i}=r$ if the $i$ th particle survives $r$ times as a type 1 particle then dies, or survives one last time as a type 2 particle then dies. Let us introduce the sequence $\left(V_{i}^{(r)}\right)_{r \in \mathbb{N}}$ representing the successive time instants of this particle (arriving at time $T_{i}$ ) changing back to type 1 after being type 2 prior to its death. In other words, this $i$ th particle becomes type 1 again at times $T_{i}+V_{i}^{(1)}, T_{i}+V_{i}^{(2)}$, etc. Then the sequence is expressed as $V_{i}^{(r)}-V_{i}^{(r-1)}=Y_{1, i}^{(r)}+Y_{2, i}^{(r)}$ from $r \in \mathbb{N}^{*}$ with $V_{i}^{(0)}=0$ where $Y_{j, i}^{(r)}$ represents the $r$ th sojourn time of type $j$ particle for $j=1,2$ such that the $\operatorname{rv} Y_{j, i}^{(r)}, j=1,2$, $i \in \mathbb{N}^{*}, r \in \mathbb{N}^{*}$, are independent, with distributions given by $\mathcal{D}\left(Y_{j, i}^{(r)}\right)=\mathcal{E}\left(\mu_{j}\right)$. See Figure 1 for an illustration. Then, $N_{1}(t)$ has the following expression

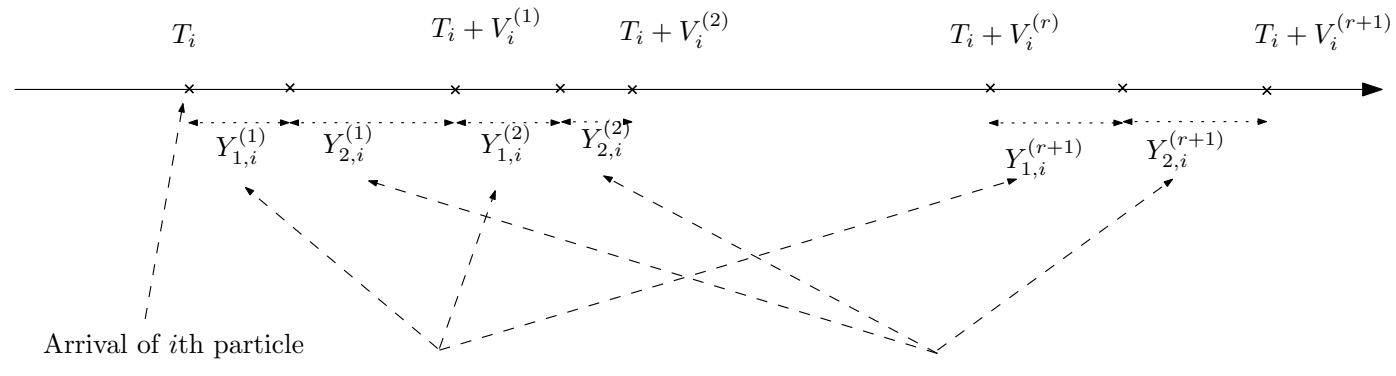

Type 1

FIG 1. Evolution of ith particle.

$$
N_{1}(t)=\sum_{i=1}^{\infty} \sum_{r=0}^{G_{i}-1} \mathbb{1}_{\left[T_{i}+V_{i}^{(r)} \leq t<T_{i}+V_{i}^{(r)}+Y_{1, i}^{(r+1)}\right]},
$$

as $\left[T_{i}+V_{i}^{(r)} \leq t<T_{i}+V_{i}^{(r)}+Y_{1, i}^{(r+1)}\right]$ corresponds to the event that type 1 particle which arrived at time $T_{i}$ is again type 1 at time $t$ after its $r$ th return time. Taking the expectation in (89), interchanging the order of summation and using the independence of $G_{i}$ from the arrival process $\{S(t), t \geq 0\}$ and $\left(Y_{j, i}^{(r)}\right)_{r \in \mathbb{N}^{*}}, j=1,2$, yield

$$
\begin{aligned}
\mathbb{E}\left[N_{1}(t)\right] & =\sum_{r=0}^{\infty} B_{r}\left(p_{12} p_{21}\right)^{r}, \\
B_{r} & :=\sum_{i=1}^{\infty} \mathbb{P}\left(T_{i}+V_{i}^{(r)} \leq t<T_{i}+V_{i}^{(r)}+Y_{1, i}^{(r+1)}\right) .
\end{aligned}
$$

Let us denote by $G^{(r)}(\cdot)$ the cumulative distribution function of $V_{i}^{(r)}$, in other words, the $r$ th convolution of the sum of two exponential variables with mean $\mu_{1}$ and mean $\mu_{2}$, and also denote $G^{(0)}(d s)=\delta_{0}(d s)$. By the independence of the arrival process $\{S(t), t \geq 0\}, V_{i}^{(r)}$ and $Y_{1, i}^{(r+1)}$ we get

$$
B_{r}=\int_{0}^{t} \int_{0}^{t}[m(t-v)-m(t-v-z)] G^{(r)}(d v) \mu_{1} e^{-\mu_{1} z} d z .
$$

It then follows from $(90)$ that $\mathbb{E}\left[N_{1}(t)\right]$ is given by

$$
\mathbb{E}\left[N_{1}(t)\right]=\int_{0}^{t} \int_{0}^{t}[m(t-v)-m(t-v-z)] \Psi(d v) \mu_{1} e^{-\mu_{1} z} d z, \quad t \geq 0,
$$


where $\Psi(d s)$ is a measure defined by

$$
\Psi(d s)=\sum_{r=0}^{\infty}\left(p_{12} p_{21}\right)^{r} G^{(r)}(d s),
$$

which remains to be determined. Since $G^{(r)}(d s)$ is the distribution of the sum of two independent Erlang distributions with respective parameters $\left(r, \mu_{1}\right)$ and $\left(r, \mu_{2}\right)$, its Laplace transform is given by

$$
\widehat{G^{(r)}}(x)=\int_{0}^{\infty} e^{-x s} G^{(r)}(d s)=\left(\frac{\mu_{1}}{\mu_{1}+x} \frac{\mu_{2}}{\mu_{2}+x}\right)^{r}, \quad r \geq 0, \quad x \geq 0,
$$

so that, taking the Laplace transform on both sides of (92), one obtains

$$
\begin{gathered}
\widehat{\Psi}(x)=\sum_{r=0}^{\infty}\left(p_{12} p_{21}\right)^{r} \widehat{G^{(r)}}(x)=\frac{1}{1-p_{12} p_{21} \frac{\mu_{1}}{\mu_{1}+x} \frac{\mu_{2}}{\mu_{2}+x}}=1+\frac{\mu_{1} \mu_{2} p_{12} p_{21}}{x^{2}+\left(\mu_{1}+\mu_{2}\right) x+\mu_{1} \mu_{2}\left(1-p_{12} p_{21}\right)} \\
=1+\frac{\mu_{1} \mu_{2} p_{12} p_{21}}{\left(x-\zeta_{1}\right)\left(x-\zeta_{2}\right)}=1+\mu_{1} \mu_{2} p_{12} p_{21}\left[\frac{1}{\left(\zeta_{1}-\zeta_{2}\right)\left(x-\zeta_{1}\right)}+\frac{1}{\left(\zeta_{2}-\zeta_{1}\right)\left(x-\zeta_{2}\right)}\right], \quad \text { (93) }
\end{gathered}
$$

where $\zeta_{1}$ and $\zeta_{2}$ are defined by (87) and (88). Inverting (93) then yields

$$
\Psi(d s)=\delta_{0}(d s)+\mu_{1} \mu_{2} p_{12} p_{21}\left[\frac{1}{\zeta_{1}\left(\zeta_{2}-\zeta_{1}\right)} e^{\zeta_{1} s}+\frac{1}{\zeta_{2}\left(\zeta_{1}-\zeta_{2}\right)} e^{\zeta_{2} s}\right] d s, s \geq 0
$$

which, by substituting in (91), yields (86).

Remark 15. Similar analysis is available to obtain a transient expression for $\mathbb{E}\left[N_{2}(t)\right]$ for type 2 particles, as well as the expected number of particles, say $\mathbb{E}\left[M_{1}(t)\right]$ of type 1 when immigration has the distribution $I \sim \delta_{(0,1)}$, i.e. when one particle of type 2 arrives at each instant $T_{i}$. By the superposition principle (3)), one deduces that, for a general immigration vector $I=(I(1), I(2))$ in (2), the total number of expected particles of type 1 is then given by

$$
\mathbb{E}(I(1)) \mathbb{E}\left[N_{1}(t)\right]+\mathbb{E}(I(2)) \mathbb{E}\left[M_{1}(t)\right]
$$

\section{References}

[1] Allison, P.D. (1980). Estimation and testing for a Markov model of reinforcement. Socialogical Methods and Research, 8(4): 434-453.

[2] Altman, E. (2005). On stochastic recursive equations and infinite server queues. Proceedings of IEEE Infocom, Miami, 13-17 March 2005.

[3] Asmussen, S. and Bladt, M. (1996). Renewal theory and queueing algorithms for matrix exponential distributions. Matrix analytic methods in stochastic models (A.S. Alsfa $\& S$ S. Chakravarty eds.): 313-341. Marcel Dekker, New York.

[4] Athreya, A.B. (1969). Limit theorems for multitype continuous time Markov branching processes, II. The case of an arbitrary linear functional. Zeitschrift für Wahrscheinlichkeitstheorie und Verwandte Gebiete, 13: 204-214.

[5] Athreya, A.B. and Ney, P.E. (1972). Branching processes. Springer-Verlag. 
[6] Badía F.G., Sangüesa C. and Cha J.H. (2018). Univariate and multivariate stochastic comparisons and ageing properties of the Generalized Pólya process. Journal of Applied Probability, 55: 233-253.

[7] Bates, G.E. (1955). Joint distributions of time intervals for the occurrence of successive accidents in a generalized Polya scheme. Annals of Mathematical Statistics, 26(4): 705720.

[8] Bühlmann, H. (1970). Mathematical methods in risk theory. Springer.

[9] Cha, J.H. (2014). Characterization of the generalized Pólya process and its applications. Advances in Applied Probability, 46: 1148-1171.

[10] Cha, J.H. and Finkelstein, M. (2016). New shock models based on the generalized Polya process. European Journal of Operational Research, 251: 135-141.

[11] Cha, J.H. and Finkelstein, M. (2016). Justifying the Gompertz curve of mortality via the generalized Polya process of shocks. Theoretical Population Biology, 109: 54-62.

[12] Durham, S.D. (1971). A problem concerning generalized age-dependent branching processes with immigration. Annals of Mathematical Statistics, 42(3): 1121-1123.

[13] Feller, W. (1943). On a general class of "contagious" distributions. Annals of Mathematical Statistics, 14(4): 389-400.

[14] Hyrien, O., Peslak, S. A., Yanev, N. M. and Palis, J. (2015). Stochastic modeling of stress erythropoiesis using a two-type age-dependent branching process with immigration. Journal of Mathematical Biology, 70: 1485-1521.

[15] Hyrien, O., Mitov, K.V., Yanev, N.M. (2016). Supercritical Sevastyanov branching processes with non-homogeneous Poisson immigration. In Branching Processes and their Applications, pages 151-166. Springer.

[16] Hyrien, O., Mitov, K.V., Yanev, N.M. (2017). Subcritical Sevastyanov branching processes with nonhomogeneous Poisson immigration. Journal of Applied Probability, 54(2):569587.

[17] Greenwood, M., Yule, G. U. (1920). An inquiry into the nature of frequency distributions representative of multiple happenings with particular reference to the occurrence of multiple attacks of disease or of repeated accidents. Journal of the Royal Statistical Society, Series A, 83: 255-279.

[18] Jeanblanc M., Yor M., Chesney M. (2009). Poisson Processes and Ruin Theory. In: Mathematical Methods for Financial Markets. Springer Finance. Springer, London.

[19] Konno, H. (2010). On the exact solution of a generalized Polya process. Advances in Mathematical Physics, 2010: Article ID 504267.

[20] Kyprianou, A.E. (2006). Introductory lectures on fluctuations of Lévy processes with applications. Springer.

[21] Landriault, D., Willmot, G.E. and Xu, D. (2014). On the analysis of time dependent claims in a class of birth process claim count models. Insurance: Mathematics and Economics, 58: 168-173.

[22] Laskin, N. (2003). Fractional Poisson process. Communications in nonlinear science and numerical simulation, 8: 201-213.

[23] Le Gat, Y. (2014). Extending the Yule process to model recurrent pipe failures in water supply networks. Urban Water Journal, 11(8): 617-630.

[24] Mitov, K.V., Yanev, N.M. and Hyrien, O. (2018). Multitype branching processes with inhomogeneous Poisson immigration. Advances in Applied Probability, 50(A):211-228.

[25] Pólya, G. (1930). Sur quelques points de la théorie des probabilités. Annales de l'Institut H. Poincaré, 1: 117-161. 
[26] Qi, J., Ju W. and Sun K. (2017). Estimating the propagation of interdependent cascading outages with multi-type branching processes. IEEE Transactions on Power Systems, 32(2):1212-1223.

[27] Resing, J.A.C. (1993). Polling systems and multitype branching processes. Queueing Systems, 13: 409-426.

[28] Rolski, D.T., Schmidli, H., Schmidt, V. and Teugels, J.L. (1998). Stochastic Processes for Insurance and Finance. Wiley.

[29] Sevast'yanov, B.A. (1971). Branching Processes. Nauka, Moscow (in Russian).

[30] van der Mei, R.D. (2007). Towards a unifying theory on branching-type polling systems in heavy traffic. Queueing Systems, 57(1): 29-46.

[31] Vatutin, V.A. (1977). A critical Bellman-Harris branching process with immigration and several types of particles. Theory of Probability and its Applications, 21(2): 435-442.

[32] Wasserman, S. (1983). Distinguishing between stochastic models of heterogeneity and contagion. Journal of Mathematical Psychology, 27: 201-215.

[33] Weiner, H.J. (1970). On a multi-type critical age-dependent branching process. Journal of Applied Probability, 7: 523-543.

[34] Weiner, H.J. (1972). A multi-type critical age-dependent branching process with immigration. Journal of Applied Probability, 9: 697-706.

[35] Willmot, G.E. (2010). Distributional analysis of a generalization of the Polya process. Insurance: Mathematics and Economics, 47: 423-427.

\section{Appendix A: A growth rate for the second order moment of $N^{o}(t)$}

Let $D(t):=\left(\mathbb{E}\left(N_{i}^{o}(t) N_{l}^{o}(t)\right)\right)_{i, l=1, \ldots, k}=\mathbb{E}\left(N^{o}(t) N^{o}(t)^{\prime}\right)$ be the second order matrix at time $t \geq$ 0 of the the baseline multitype branching process $\left\{N^{o}(t), t \geq 0\right\}$, with branching mechanism in (1) and immigration vector at time 0 in (2) described in Section 2. The following lemma is a consequence of the growth rates for the second moments provided in [5, Section 7.4 p.203] which are the results obtained when $N^{o}(0)$ is deterministic.

Lemma 16. The following growth rate holds entrywise under Assumption (A) as $t \rightarrow \infty$ :

$$
D(t)=\left\{\begin{aligned}
O\left(e^{\rho t}\right) & \text { if } \rho<0 \\
O(t) & \text { if } \rho=0 \\
O\left(e^{2 \rho t}\right) & \text { if } \rho>0
\end{aligned}\right.
$$

Proof. Let us denote $D\left(t \mid \mathbf{n}_{0}\right):=\mathbb{E}\left(N^{o}(t) N^{o}(t)^{\prime} \mid N^{o}(0)=\mathbf{n}_{0}\right)$ with $\mathbf{n}_{0}=\left(\mathbf{n}_{0}(1), \ldots, \mathbf{n}_{0}(k)\right) \in$ $\mathbb{N}^{k}$. The superposition principle (3) and the independence of processes $\left(\left\{N^{j, l}(t), t \geq 0\right\}\right)_{j, l \in \mathbb{N}^{2}}$ entails that

$$
\begin{aligned}
D\left(t \mid \mathbf{n}_{0}\right)= & \sum_{j=1}^{k} \sum_{l=1}^{\mathbf{n}_{0}(j)} \mathbb{E}\left(N^{j, l}(t) N^{j, l}(t)^{\prime}\right)+\sum_{j=1}^{k} \sum_{\substack{l, r=1, \ldots, \mathbf{n}_{0}(j) \\
l \neq r}} \mathbb{E}\left(N^{j, l}\right) \mathbb{E}\left(N^{j, r}\right)^{\prime} \\
& +\sum_{\substack{j, p=1, \ldots, k, j \neq p}} \sum_{\substack{l=1, \ldots, \mathbf{n}_{0}(j), r=1, \ldots, \mathbf{n}_{0}(t)}} \mathbb{E}\left(N^{j, l}(t)\right) \mathbb{E}\left(N^{p, r}(t)\right)^{\prime} \\
= & \sum_{j=1}^{k} \mathbf{n}_{0}(j) \mathbb{E}\left(N^{j}(t) N^{j}(t)^{\prime}\right)+\sum_{j=1}^{k}\left(\mathbf{n}_{0}(j)-1\right) \mathbf{n}_{0}(j) \mathbb{E}\left(N^{j}(t)\right) \mathbb{E}\left(N^{j}(t)\right)^{\prime}
\end{aligned}
$$




$$
+\sum_{\substack{j, p=1, \ldots, k, j \neq p}} \mathbf{n}_{0}(j) \mathbf{n}_{0}(p) \mathbb{E}\left(N^{j}(t)\right) \mathbb{E}\left(N^{p}(t)\right)^{\prime} .
$$

We study the terms on the right-hand side of (95) in the following cases for the eigenvalue $\rho$, yielding the growth rates (94).

If $\rho<0$, we have from [5, (19) p.204] that $\mathbb{E}\left(N^{j}(t) N^{j}(t)^{\prime}\right)=O\left(e^{\rho t}\right)$ entrywise and uniformly in all $j=1, \ldots, k$, and from $\left[5,(17)\right.$ p.203] that $\mathbb{E}\left(N^{j}(t)\right)=O\left(e^{\rho t}\right)$ entrywise and uniformly in all $j=1, \ldots, k$. The latter estimate yields that $\mathbb{E}\left(N^{j}(t)\right) \mathbb{E}\left(N^{j}(t)\right)^{\prime}$ and $\mathbb{E}\left(N^{j}(t)\right) \mathbb{E}\left(N^{p}(t)\right)^{\prime}$ are $O\left(e^{2 \rho t}\right)$, hence also $O\left(e^{\rho t}\right)$ (as $\rho<0$ ) entrywise uniformly in all $j, p=1, \ldots, k$, yielding in turn from (95) that

$$
D\left(t \mid \mathbf{n}_{0}\right)=\left[\sum_{j=1}^{k} \mathbf{n}_{0}(j)^{2}\right] O\left(e^{\rho t}\right),
$$

meaning that each entry of $D^{\left(\mathbf{n}_{0}\right)}(t)$ grows at most as $\left[\sum_{j=1}^{k} \mathbf{n}_{0}(j)^{2}\right] C e^{\rho t}$ for some constant $C$ independent from $\mathbf{n}_{0}$. This finally implies, thanks to Assumption (A), that

$$
D(t)=\sum_{\mathbf{n}_{0} \in \mathbb{N}^{k}} D\left(t \mid \mathbf{n}_{0}\right) p_{\mathbf{n}_{0}}=\sum_{\mathbf{n} \in \mathbb{N}^{k}}\left[\sum_{j=1}^{k} \mathbf{n}_{0}(j)^{2}\right] p_{\mathbf{n}_{0}} O\left(e^{\rho t}\right)=\mathbb{E}\left(\|I\|^{2}\right) O\left(e^{\rho t}\right)=O\left(e^{\rho t}\right),
$$

proving (94) when $\rho<0$. If $\rho=0$, we have from [5, (19) p.204] that $\mathbb{E}\left(N^{j}(t) N^{j}(t)^{\prime}\right)=O(t)$ entrywise and uniformly in all $j=1, \ldots, k$, and from [5, (17) p.203] that $\mathbb{E}\left(N^{j}(t)\right)=O(1)$ entrywise and uniformly in all $j=1, \ldots, k$. Thus similar analysis using (95) and Assumption (A) implies the growth rate (94) when $\rho=0$. Finally, if $\rho=0$, we have from [5, (19) p.204] that $\mathbb{E}\left(N^{j}(t) N^{j}(t)^{\prime}\right)=O\left(e^{2 \rho t}\right)$ entrywise and uniformly in all $j=1, \ldots, k$, and from [5, (17) p.203] that $\mathbb{E}\left(N^{j}(t)\right)=O\left(e^{\rho t}\right)$ entrywise and uniformly in all $j=1, \ldots, k$, implying in turn that $\mathbb{E}\left(N^{j}(t)\right) \mathbb{E}\left(N^{j}(t)\right)^{\prime}$ and $\mathbb{E}\left(N^{j}(t)\right) \mathbb{E}\left(N^{p}(t)\right)^{\prime}$ are $O\left(e^{2 \rho t}\right)$. Similar analysis implies the growth rate (94) when $\rho>0$.

\section{Appendix B: Proof of Lemma 8}

Before proceeding with the proof, we recall the multidimensional version of Polya's theorem, which will be used later on.

Lemma 17. Let $\left\{X_{t}, t \geq 0\right\}$ be a sequence of random variables with values in $\mathbb{R}^{k}$ converging in distribution towards $X \in \mathbb{R}^{k}$, such that $x \in \mathbb{R}^{k} \mapsto \mathbb{P}(X \leq x)$ is continuous. Then one has for all $x \in \mathbb{R}^{k}$ that

$$
\lim _{t \rightarrow+\infty} \mathbb{P}\left(X_{t}>x_{t}\right)=\mathbb{P}(X>x)
$$

where $\lim _{t \rightarrow \infty} x_{t}=x, x_{t}$ lying in $\mathbb{R}^{k}$, and ' $\leq$ ' and ' $>$ ' are understood componentwise.

We now turn to the proof of Lemma 8. First, for the process $N^{j}(t)=\left(N_{1}^{j}(t), \ldots, N_{k}^{j}(t)\right)$ described in Section 2 one has that

$$
\exp \left(i s_{l} N_{l}^{j}\left(t-\Lambda^{-1}(\Lambda(t) x)\right) / t\right)=\int_{\mathbb{R}_{+}^{*}} i s_{l} \exp \left(i s_{l} z_{l}\right) \mathbb{1}_{\left[z_{l}<N_{l}^{j}\left(t-\Lambda^{-1}(\Lambda(t) x)\right) / t\right]} d z_{l}+1, \quad \forall s_{l} \in \mathbb{R},
$$

for $l=1, \ldots, k$. Together with an expansion formula, we get that 


$$
\begin{array}{r}
\exp \left(<i s, \frac{N^{j}\left(t-\Lambda^{-1}(\Lambda(t) x)\right)}{t}>\right)=\prod_{l=1}^{k}\left[\int_{\mathbb{R}_{+}^{*}} i s_{l} \exp \left(i s_{l} z_{l}\right) \mathbb{1}_{\left[z_{l}<N_{l}^{j}\left(t-\Lambda^{-1}(\Lambda(t) x)\right) / t\right]} d z_{l}+1\right] \\
=1+\sum_{J \subset\{1, \ldots, k\}} \int_{\left(\mathbb{R}_{+}^{*}\right)} \prod^{\operatorname{Card}(J)} \prod_{l \in J}\left[i s_{l} \exp \left(i s_{l} z_{l}\right) \mathbb{1}_{\left[z_{l}<N_{l}^{j}\left(t-\Lambda^{-1}(\Lambda(t) x)\right) / t\right]} d z_{l}\right], \quad(96)
\end{array}
$$

where $\sum_{J \subset\{1, \ldots, k\}}$ is the sum over nonempty sets $J \subset\{1, \ldots, k\}$. The results in [33] will be repeatedly used in the following leading to the convergence (47). Taking expectations on both sides of $(96)$, it follows that $\psi^{j}(t, x)$ in (50) may be expressed as

$$
\psi^{j}(t, x)-1=\sum_{J \subset\{1, \ldots, k\}} \int_{\left(\mathbb{R}_{+}^{*}\right)^{\operatorname{Card}(J)}} \prod_{l \in J}\left[i s_{l} \exp \left(i s_{l} z_{l}\right) \mathbb{P}\left(z_{l}<N_{l}^{j}\left(t-\Lambda^{-1}(\Lambda(t) x)\right) / t\right) d z_{l}\right],
$$

which, using the notation $z^{J}$ introduced just before Lemma 8 and multiplied by $t$, drives a more compact form given by

$$
\begin{aligned}
t\left[\psi^{j}(t, x)-1\right]= & \sum_{J \subset\{1, \ldots, k\}} \\
& \int_{\left(\mathbb{R}_{+}^{*}\right)} \prod^{\operatorname{Card}(J)} \prod_{l \in J}\left[i s_{l} \exp \left(i s_{l} z_{l}\right)\right] t \mathbb{P}\left(N^{j}\left(t-\Lambda^{-1}(\Lambda(t) x)\right) / t>z^{J}\right) d z^{J},
\end{aligned}
$$

where, for two vectors $v_{1}$ and $v_{2}, v_{1}>v_{2}$ means that each entry of $v_{1}$ is larger than the corresponding one in $v_{2}$, and $d z^{J}:=\prod_{l \in J} d z_{l}$.

Next, let us observe that, for a fixed $x \in(0,1)$, from (42) it follows that $t-\Lambda^{-1}(\Lambda(t) x)=$ $t-\lambda_{\infty}^{-1} \Lambda(t) x-\eta(\Lambda(t) x) \Lambda(t) x=t-\lambda_{\infty}^{-1} \Lambda(t) x+o(t)$ and also $\lambda_{\infty}^{-1} \Lambda(t) x=\lambda_{\infty}^{-1}\left[\lambda_{\infty} t+o(t)\right] x=$ $t x+o(t)$ due to $\lim _{t \rightarrow+\infty} \Lambda(t) / t=\lambda_{\infty}$. Thus, one finds that

$$
t-\Lambda^{-1}(\Lambda(t) x) \sim t(1-x), \quad t \rightarrow+\infty .
$$

Since the above result entails that $t-\Lambda^{-1}(\Lambda(t) x) \longrightarrow+\infty$ as $t \rightarrow+\infty$, from [33, Theorems 1 and 5$]$, we find for $x \in(0,1)$ that

$$
\begin{aligned}
& {\left[t-\Lambda^{-1}(\Lambda(t) x)\right] \mathbb{P}\left(N^{j}\left(t-\Lambda^{-1}(\Lambda(t) x)\right)>0\right) \longrightarrow \beta_{j},} \\
& \mathbb{P}\left(\frac{N^{j}\left(t-\Lambda^{-1}(\Lambda(t) x)\right)}{t-\Lambda^{-1}(\Lambda(t) x)}>z \mid N^{j}\left(t-\Lambda^{-1}(\Lambda(t) x)\right)>0\right) \rightarrow \exp \left(-c \max _{i=1, \ldots, k} \frac{z_{i}}{v_{i} \mu_{i}^{-1}}\right) \\
& =\mathbb{P}(\mathcal{X}>z),
\end{aligned}
$$

as $t \rightarrow+\infty$ and for all $z=\left(z_{1}, \ldots, z_{k}\right) \in \mathbb{R}_{+}^{* k}$, where we recall that $\beta_{j}$ is given by (13) and that $\mathcal{X}$ has a distribution given by (45). Here again, the relation ' $>$ ' is understood entrywise. It is noted that (100) simply states that the distribution of $\frac{N^{j}\left(t-\Lambda^{-1}(\Lambda(t) x)\right)}{t-\Lambda^{-1}(\Lambda(t) x)}$ given that $N^{j}(t-$ $\left.\Lambda^{-1}(\Lambda(t) x)\right)>0$ converges to the distribution of $\mathcal{X}$. Also, since $z \in \mathbb{R}^{k} \mapsto \mathbb{P}(\mathcal{X}>z)$ is continuous (extending the definition in (45) from $z \in \mathbb{R}_{+}^{* k}$ to $z \in \mathbb{R}^{k}$ by putting $\mathbb{P}(\mathcal{X}>z)=1$ if $\left.\max _{i=1, \ldots, k} z_{i} \leq 0\right)$, and $\lim _{t \rightarrow+\infty} \frac{t}{t-\Lambda^{-1}(\Lambda(t) x)}=\frac{1}{1-x}$ from (98), one has from Lemma 17 for all $z$ that

$$
\mathbb{P}\left(\frac{N^{j}\left(t-\Lambda^{-1}(\Lambda(t) x)\right)}{t}>z \mid N^{j}\left(t-\Lambda^{-1}(\Lambda(t) x)\right)>0\right)
$$




$$
\begin{aligned}
=\mathbb{P}\left(\frac{N^{j}\left(t-\Lambda^{-1}(\Lambda(t) x)\right)}{t-\Lambda^{-1}(\Lambda(t) x)}\right. & \left.>\frac{t}{t-\Lambda^{-1}(\Lambda(t) x)} z \mid N^{j}\left(t-\Lambda^{-1}(\Lambda(t) x)\right)>0\right) \\
& \longrightarrow \mathbb{P}\left(\mathcal{X}>\frac{1}{1-x} z\right)=\mathbb{P}((1-x) \mathcal{X}>z), \quad t \rightarrow+\infty
\end{aligned}
$$

for a fixed $x \in(0,1)$. The latter convergence along with (98) and (99) entail that the components of the integrand in (97) satisfy

$$
\begin{aligned}
t \mathbb{P}\left(\frac{N^{j}\left(t-\Lambda^{-1}(\Lambda(t) x)\right)}{t}>z^{J}\right) & \\
=\frac{t}{t-\Lambda^{-1}(\Lambda(t) x)} & \mathbb{P}\left(\frac{N^{j}\left(t-\Lambda^{-1}(\Lambda(t) x)\right)}{t}>z^{J} \mid N^{j}\left(t-\Lambda^{-1}(\Lambda(t) x)\right)>0\right) \\
\times[t & \left.-\Lambda^{-1}(\Lambda(t) x)\right] \mathbb{P}\left(N^{j}\left(t-\Lambda^{-1}(\Lambda(t) x)\right)>0\right) \\
& \longrightarrow \frac{1}{1-x} \beta_{j} \mathbb{P}\left((1-x) \mathcal{X}>z^{J}\right), \quad t \rightarrow+\infty, \quad \forall J \subset\{1, \ldots, k\} .
\end{aligned}
$$

It is important to note that from the convergence result in (99), $\left[t-\Lambda^{-1}(\Lambda(t) x)\right] \mathbb{P}\left(N^{o}(t-\right.$ $\left.\Lambda^{-1}(\Lambda(t) x)\right)>0$ ) is bounded uniformly in $t \geq 0$ and $x \in(0,1)$ by some constant. Furthermore, (100) says that $\mathcal{A}_{t}$ converges in distribution towards $(1-x) \mathcal{X}$ as $t \rightarrow \infty$, where $\mathcal{A}_{t}$ is a random vector such that $\mathcal{D}\left(\mathcal{A}_{t}\right)=\mathcal{D}\left(\frac{N^{j}\left(t-\Lambda^{-1}(\Lambda(t) x)\right)}{t} \mid N^{j}\left(t-\Lambda^{-1}(\Lambda(t) x)\right)>0\right)$ for all $t \geq 0$. We recall that $\mathcal{X}$ admits the expression (44), and we thus deduce that $(1-x) \mathcal{X}$ is light tailed, i.e. that there exists some vector $w_{0} \in \mathbb{R}^{k}$ close to 0 with positive entries such that $\mathbb{E}(\exp (<$ $\left.\left.(1-x) \mathcal{X}, w_{0}>\right)\right)$ is finite. The fact that $w_{0}$ has positive entries together with Chernoff's bound thus yields that

$$
\begin{aligned}
& \mathbb{P}\left(\frac{N^{j}\left(t-\Lambda^{-1}(\Lambda(t) x)\right)}{t}>z^{J} \mid N^{j}\left(t-\Lambda^{-1}(\Lambda(t) x)\right)>0\right)=\mathbb{P}\left(\mathcal{A}_{t}>z^{J}\right) \\
\leq & \mathbb{P}\left(<\mathcal{A}_{t}, w_{0}>><z^{J}, w_{0}>\right) \leq \mathbb{E}\left(\exp \left(<\mathcal{A}_{t}, w_{0}>\right)\right) e^{-<z^{J}, w_{0}>} \\
\longrightarrow & \mathbb{E}\left(\exp \left(<(1-x) \mathcal{X}, w_{0}>\right)\right) e^{-<z^{J}, w_{0}>}, \quad t \rightarrow \infty .
\end{aligned}
$$

Also, $\frac{t}{t-\Lambda^{-1}(\Lambda(t) x)}$ is upper bounded in $t \geq 0$ by some constant that depends on $x$ as it is convergent towards $\frac{1}{1-x}$ as $t \rightarrow+\infty$. Therefore, from the above findings we conclude that the following function is bounded by

$$
t \mathbb{P}\left(\frac{N^{j}\left(t-\Lambda^{-1}(\Lambda(t) x)\right)}{t}>z^{J}\right) \leq K_{x}^{j} e^{-<z^{J}, w_{0}>}, \quad \forall J \subset\{1, \ldots, k\}, \quad \forall t \geq 0,
$$

where $K_{x}^{j}$ is some constant independent from $t \geq 0$ and $z \in \mathbb{R}_{+}^{* k}$. Since $\int_{\left(\mathbb{R}_{+}^{*}\right)^{\operatorname{Card}(J)}} e^{-<z^{J}, w_{0}>} d z^{J}$ is finite for all $J \subset\{1, \ldots, k\}$, one finds by the dominated convergence theorem that the integrand in (97) satisfies

$$
\begin{aligned}
& \int_{\left(\mathbb{R}_{+}^{*}\right)^{\operatorname{Card}(J)}} \prod_{l \in J}\left[i s_{l} \exp \left(i s_{l} z_{l}\right)\right] t \mathbb{P}\left(N^{j}\left(t-\Lambda^{-1}(\Lambda(t) x)\right) / t>z^{J}\right) d z^{J} \\
& \longrightarrow \int_{\left(\mathbb{R}_{+}^{*}\right)^{\operatorname{Card}(J)}} \prod_{l \in J}\left[i s_{l} \exp \left(i s_{l} z_{l}\right)\right]\left\{\frac{1}{1-x} \beta_{j} \mathbb{P}\left((1-x) \mathcal{X}>z^{J}\right)\right\} d z^{J}, \quad t \rightarrow+\infty, \quad \forall J \subset\{1, \ldots, k\}
\end{aligned}
$$


for a fixed $x \in(0,1)$. Putting this into (97) yields the following limit as $t \rightarrow \infty$

$$
t\left[\psi^{j}(t, x)-1\right] \longrightarrow \sum_{J \subset\{1, \ldots, k\}} \int_{\left(\mathbb{R}_{+}^{*}\right) \operatorname{Card}(J)} \prod_{l \in J}\left[i s_{l} \exp \left(i s_{l} z_{l}\right)\right]\left\{\frac{1}{1-x} \beta_{j} \mathbb{P}\left((1-x) \mathcal{X}>z^{J}\right)\right\} d z^{J}
$$

By an expansion formula similar to $(96)$ (with $(1-x) \mathcal{X}$ here instead of $\left.N^{j}\left(t-\Lambda^{-1}(\Lambda(t) x)\right) / t\right)$, one can check that the right-hand side of the above limit is equal to $\frac{1}{1-x} \beta_{j}[1-\mathbb{E}(\exp [<s,(1-x) \mathcal{X}>])]$, yielding (51).

\section{Appendix C: Proof of Lemma 13}

The moment generating function of $T \sim \Gamma(\zeta, 1)$ is given by $\mathbb{E}\left(e^{z T}\right)=(1-z)^{-\zeta}$ for $z$ being a complex number with real part less than 1 . By the independence assumption, one then gets

$$
\mathbb{E}\left[e^{i x \mathcal{Z}_{T}}\right]=\int_{0}^{\infty} \mathbb{E}\left[e^{i x \mathcal{Z}_{t}}\right] \mathbb{P}(T \in d t)=\int_{0}^{\infty} e^{-t \psi(x)} \mathbb{P}(T \in d t)=\{1+\psi(x)\}^{-\zeta},
$$

which completes the proof. 